\title{
Nilsson solutions for irregular $A$-hypergeometric systems
}

\author{
Alicia Dickenstein, Federico N. Martínez, Laura Felicia Matusevich
}

\begin{abstract}
We study the solutions of irregular $A$-hypergeometric systems that are constructed from Gröbner degenerations with respect to generic positive weight vectors. These are formal logarithmic Puiseux series that belong to explicitly described Nilsson rings, and are therefore called (formal) Nilsson series. When the weight vector is a perturbation of $(1, \ldots, 1)$, these series converge and provide a basis for the (multivalued) holomorphic hypergeometric functions in a specific open subset of $\mathbb{C}^{n}$. Our results are more explicit when the parameters are generic or when the solutions studied are logarithm-free. We also give an alternative proof of a result of Schulze and Walther that inhomogeneous $A$-hypergeometric systems have irregular singularities.
\end{abstract}

\section{Introduction}

The Frobenius method is a symbolic procedure for solving a linear ordinary differential equation in a neighborhood of a regular singular point. The solutions are represented as convergent logarithmic Puiseux series that belong to the Nilsson class. In the multivariate case, a direct generalization of the Frobenius method, called the canonical series algorithm, was introduced by Saito, Sturmfels and Takayama in Chapter 2.5 of [24], based on Gröbner degenerations in the Weyl algebra $D$. When applied to a regular holonomic left $D$-ideal, this procedure yields a basis of the solution space. The basis elements belong to an explicitly described Nilsson ring, and are therefore called Nilsson series, or Nilsson solutions. Each Nilsson ring is constructed using a weight vector; the choice of weight vector is a way of determining the common domain of convergence of the corresponding solutions. The canonical series procedure requires a regular holonomic input; although one can run this algorithm on holonomic left $D$-ideals that have irregular singularities,

Mathematics Subject Classification (2010): 33C70, 14F10, 14M25, 32C38.

Keywords: A-hypergeometric functions, irregular holonomic $D$-modules, formal Nilsson series, Gröbner degenerations in the Weyl algebra. 
there is no guarantee that the output series converge, or even that the correct number of basis elements will be produced.

In this article, we study the solutions of the $A$-hypergeometric systems introduced by Gel'fand, Graev, Kapranov and Zelevinsky [11], [13].

Definition 1.1. Denote by $D$ the Weyl algebra on $x_{1}, \ldots, x_{n}$ and $\partial_{1}, \ldots, \partial_{n}$, where $\partial_{i}$ stands for the partial derivative with respect to $x_{i}$. Let $A=\left[a_{i j}\right] \in \mathbb{Z}^{d \times n}$ be such that its rows $\mathbb{Z}$-span $\mathbb{Z}^{d}$, and let $\beta \in \mathbb{C}^{d}$. The A-hypergeometric system with parameter $\beta$ is the left $D$-ideal

$$
H_{A}(\beta)=I_{A}+\left\langle E_{1}-\beta_{1}, \ldots, E_{d}-\beta_{d}\right\rangle \subset D,
$$

where $E_{i}=\sum_{j=1}^{n} a_{i j} x_{j} \partial_{j}, 1 \leq i \leq d$, and $I_{A}$ denotes the toric ideal

$$
I_{A}=\left\langle\partial^{u}-\partial^{v} \mid A \cdot u=A \cdot v\right\rangle \subseteq \mathbb{C}[\partial] .
$$

These left $D$-ideals are always holonomic [1], [13]. It is known that $H_{A}(\beta)$ is regular holonomic if and only if the $\mathbb{Q}$-rowspan of the matrix $A$ contains the vector $(1, \ldots, 1)$. The if direction was proved by Hotta in his work on equivariant $D$-modules [16]; Saito, Sturmfels and Takayama gave a partial converse, see Theorem 2.4.11 in [24], where it is assumed that the parameter $\beta$ is generic. Their proof uses Gröbner methods in $D$.

A different strategy to show that a $D$-ideal is not regular holonomic is to prove that it has slopes. The analytic slopes of a $D$-module were introduced by Mebkhout in [20], while an algebraic version was given by Laurent [17]. These authors have shown that the analytic and algebraic slopes of a $D$-module along a smooth hypersurface agree [18]. From a computational perspective, Assi, Castro-Jiménez and Granger gave a Gröbner basis algorithm to find algebraic slopes [2]. There has been an effort to compute the (algebraic) slopes of $H_{A}(\beta)$ along a coordinate hypersurface. In the cases $d=1$ and $n-d=1$, these slopes were determined by Castro-Jiménez and Takayama [7], and Hartillo-Hermoso [14], [15]. More generally, Schulze and Walther [25] have calculated the slopes of $H_{A}(\beta)$ under the assumption that the cone over the columns of $A$ contains no lines. Such a cone is called strongly convex. The fact that slopes of $H_{A}(\beta)$ always exist when the vector $(1, \ldots, 1)$ does not belong to the rowspan of $A$, implies that $H_{A}(\beta)$ has irregular singularities. Thus, Corollary 3.16 in [25] gives a converse for Hotta's regularity theorem in the strongly convex case. We give an alternative proof of this converse here, by extending ideas of Saito, Sturmfels and Takayama. The main technical obstacle to overcome is the potential existence of logarithmic hypergeometric series.

Further insight into the solutions of hypergeometric system comes from the analytic approach taken by Castro-Jiménez and Fernández-Fernández [6], [10], who studied the Gevrey filtration on the irregularity complex of an $A$-hypergeometric system. Since formal series solutions of irregular systems need not converge, a study of the Gevrey filtration provides information on how far such series are from convergence.

Even though the regularity of $H_{A}(\beta)$ is independent of $\beta$, in practice, assuming that the parameters are generic makes a difference. In this case, Ohara and 
Takayama [22] show that the method of canonical series for a weight vector which is a perturbation of $(1, \ldots, 1)$ produces a basis for the solution space of $H_{A}(\beta)$ consisting of (convergent) Nilsson series that contain no logarithms. In that work (and many others, such as [1], [19] and [24]) an important role is played by a related hypergeometric system that does have regular singularities, called the homogenized system. One of our main goals is to precisely explain the relationship between the solutions of $H_{A}(\beta)$ and those of its homogenization.

In producing a basis of solutions of $H_{A}(\beta)$ when $\beta$ is not generic, logarithmic series cannot be avoided, even in the regular case. Dealing with logarithmic solutions of $H_{A}(\beta)$ poses technical challenges that we resolve here, allowing us to lift the genericity hypotheses from the results of Ohara and Takayama: running the canonical series algorithm on $H_{A}(\beta)$ with weight vector (a perturbation of) $(1, \ldots, 1)$ always produces a basis of (convergent) Nilsson solutions of $H_{A}(\beta)$, if the cone spanned by the columns of $A$ is strongly convex. On the other hand, formal solutions of irregular hypergeometric systems that are not Nilsson series need to be considered, even in one variable (see, for instance, [9]).

This article is organized as follows. In Section 2 we introduce the formal basic Nilsson solutions of $H_{A}(\beta)$ in the direction of a weight vector $w$ (Definition 2.6), whose linear span is denoted by $\mathcal{N}_{w}\left(H_{A}(\beta)\right)$. Section 3 explains the relationship between $\mathcal{N}_{w}\left(H_{A}(\beta)\right)$ and the solution space of an associated regular holonomic hypergeometric system. A linear map $\rho$ between these spaces is constructed in Definition 3.16 and Proposition 3.17. The main result in Section 3 is Theorem 3.18, which states that $\rho$ is injective, and describes its image.

In Section 4 we restrict our attention to generic parameter vectors $\beta$, and give a combinatorial formula for the dimension of $\mathcal{N}_{w}\left(H_{A}(\beta)\right)$ (Theorem 4.9). This formula is reminiscent of the multiplicities of the $L$-characteristic cycles of the $A$-hypergeometric $D$-module $D / H_{A}(\beta)$ computed in [25], but note that these authors use different weights in the Weyl algebra than we do: while we focus on weight vectors of the form $(-w, w)$, Schulze and Walther work with $(u, v)$ such that $u+v$ is a positive multiple of $(1, \ldots, 1)$.

In Section 5 we lift the genericity assumption from $\beta$, but study only $A$-hypergeometric Nilsson series that contain no logarithms, and show that they arise from logarithm-free solutions of the associated regular holonomic hypergeometric system (Theorem 5.5). We show in Theorem 5.4 that the logarithm-free basic Nilsson solutions of $H_{A}(\beta)$ in the direction of a weight vector $w$ span the vector space of formal logarithm-free $A$-hypergeometric series in the direction of $w$, proving the formula in Display (7) in [23].

In Section 6 we analyze the convergence of formal Nilsson solutions of $A$-hypergeometric systems. The main result of that section is Theorem 6.4 , which provides an explicit construction for a basis of the space of (multivalued) holomorphic solutions of $H_{A}(\beta)$ that converge in a specific fixed open set, assuming that the cone spanned by the columns of $A$ is strongly convex. While this was well known in the regular case, it is a new result in general. Finally, under the same strong convexity assumption for $A$, we use our study of formal $A$-hypergeometric Nilsson series in Section 7 to (re)prove that $A$-hypergeometric systems arising from inhomogeneous toric ideals have irregular singularities for all parameters (Theorem 7.6). 
Acknowledgements. We are very grateful to Christine Berkesch, who proved Theorem 7.5 at our request. Part of this work was performed while the third author was visiting MSRI in the Fall 2009, and while the three authors met at the National Algebra Meeting in La Falda, Argentina, in August 2008. We thank the organizers of these events for the wonderful research atmosphere and hospitality. We are very grateful to the anonymous referee, whose thoughtful suggestions have improved the article.

\section{Initial ideals and formal Nilsson series}

Throughout this article, $A$ is a fixed $d \times n$ integer matrix whose columns $\mathbb{Z}$-span the lattice $\mathbb{Z}^{d}$. Given $\beta \in \mathbb{C}^{d}$, we consider the $A$-hypergeometric system $H_{A}(\beta)$ introduced in Definition 1.1, and we define and study its Nilsson series solutions associated to a weight vector $w \in \mathbb{R}_{\geq 0}^{n}$. We use the convention that $\mathbb{N}=\{0,1,2, \ldots\}$.

We work in the Weyl algebra $D$ in $x_{1}, \ldots, x_{n}, \partial_{1}, \ldots, \partial_{n}$. A left $D$-ideal $I$ is said to be holonomic if $\operatorname{Ext}_{D}^{i}(D / I, D)=0$ for all $i \neq n$. In this case, the holonomic rank of $I$, denoted by $\operatorname{rank}(I)$, which is by definition the dimension of the space of germs of holomorphic solutions of $I$ near a generic nonsingular point, is finite, by a result of Kashiwara (see Theorem 1.4.19 in [24]).

Definition 2.1. Let $w \in \mathbb{R}^{n}$. The $(-w, w)$-weight of $x^{u} \partial^{v} \in D$ is $-w \cdot u+w \cdot v$.

This weight induces a partial order on the monomials in the Weyl algebra.

Definition 2.2. Let $w \in \mathbb{R}^{n}$ and $f=\sum_{u, v} c_{u v} x^{u} \partial^{v} \in D$. The initial form $\operatorname{in}_{(-w, w)}(f)$ of $f \neq 0$ with respect to $(-w, w)$ is the subsum of $f$ consisting of its (nonzero) terms of maximal $(-w, w)$-weight. If $I$ is a left $D$-ideal, its initial ideal with respect to $(-w, w)$ is

$$
\operatorname{in}_{(-w, w)}(I)=\left\langle\operatorname{in}_{(-w, w)}(f) \mid f \in I, f \neq 0\right\rangle \subset D .
$$

Remark 2.3. We can restrict the $(-w, w)$-weight to the monomials in $\mathbb{C}[\partial] \subset D$; the weight of $\partial^{v}$ reduces to $w \cdot v$. When $w \in \mathbb{R}_{>0}^{n}$, the induced partial order on the monomials in $\mathbb{C}[\partial]$ has 1 as the unique smallest monomial. If $J \subseteq \mathbb{C}[\partial]$ is an ideal, we define the initial ideal $\operatorname{in}_{w}(J)$ as the ideal generated by the initial terms of all non zero polynomials in $J$.

The following definition characterizes the weight vectors we consider in this article.

Definition 2.4. A vector $w \in \mathbb{R}_{>0}^{n}$ is a weight vector for $H_{A}(\beta)$ if there exists a strongly convex open rational polyhedral cone $\mathscr{C} \subset \mathbb{R}_{>0}^{n}$, with $w \in \mathscr{C}$, such that, for all $w^{\prime} \in \mathscr{C}$, we have

$$
\operatorname{in}_{w}\left(I_{A}\right)=\operatorname{in}_{w^{\prime}}\left(I_{A}\right) \quad \text { and } \quad \operatorname{in}_{(-w, w)}\left(H_{A}(\beta)\right)=\operatorname{in}_{\left(-w^{\prime}, w^{\prime}\right)}\left(H_{A}(\beta)\right) .
$$


Note that the cone $\mathscr{C}$ is not unique and that the assumptions on $w$ in Definition 2.4 imply that $\operatorname{in}_{w}\left(I_{A}\right)$ is a monomial ideal. It follows from the existence of the Gröbner fan (see [21] for the commutative version, and [3] for the situation in the Weyl algebra) that weight vectors form an open dense subset of $\mathbb{R}_{>0}^{n}$. For an introduction to the theory of Gröbner bases in the Weyl algebra, we refer to [24]; this text is also our main reference for background on $D$-modules and hypergeometric differential equations.

Remark 2.5. Define the $A$-grading on $D$ via $\operatorname{deg}_{A}\left(x^{u} \partial^{v}\right)=A \cdot(v-u)$. A left $D$-ideal is $A$-homogeneous if it is generated by $A$-homogeneous elements of $D$. Note that $H_{A}(\beta)$ is $A$-homogeneous.

Suppose that the cone spanned by the columns of $A$ is strongly convex, that is, there exists $h \in \mathbb{R}^{d}$ such that the vector $h \cdot A$ is coordinatewise positive. Let $J$ be an $A$-homogeneous left $D$-ideal. Then for any $w \in \mathbb{R}^{n}$ there exists $w^{\prime} \in \mathbb{R}_{>0}^{n}$ such that $\operatorname{in}_{(-w, w)}(J)=\operatorname{in}_{\left(-w^{\prime}, w^{\prime}\right)}(J)$. In fact, given $w \in \mathbb{R}^{n}$, it is enough to choose a positive number $\lambda$ such that $w^{\prime}=w+\lambda h \cdot A$ is coordinatewise positive. To see this, note that if $f=\sum c_{u v} x^{u} \partial^{v} \in D$ is $A$-homogeneous, the vector $A \cdot(v-u)$ is independent of $(u, v)$ for all $c_{u v} \neq 0$. As

$$
-w^{\prime} \cdot u+w^{\prime} \cdot v=-w \cdot u+w \cdot v+\lambda[h \cdot A \cdot(v-u)]
$$

using $w^{\prime}$ instead of $w$ simply adds a constant to the weights of the terms in $f$, and then $\operatorname{in}_{(-w, w)}(f)=\operatorname{in}_{\left(-w^{\prime}, w^{\prime}\right)}(f)$. Thus, when $A$ is strongly convex, we can drop the positivity assumption in Definition 2.4.

An important special case in which the cone spanned by the columns of $A$ is strongly convex is when $(1, \ldots, 1)$ belongs to the $\mathbb{Q}$-rowspan of $A$. This happens if and only if the toric ideal $I_{A}$ is homogeneous with respect to the usual $\mathbb{Z}$-grading of the polynomial ring $\mathbb{C}[\partial]$ given by $\operatorname{deg}\left(\partial_{1}\right)=\cdots=\operatorname{deg}\left(\partial_{n}\right)=1$. We use the convention that the word homogeneous without qualifications refers to the usual $\mathbb{Z}$-grading of $\mathbb{C}[\partial]$.

If $w$ is a weight vector for $H_{A}(\beta)$, and $\mathscr{C}$ is an open cone as in Definition 2.4, the dual cone $\mathscr{C}^{*}$ consisting of elements $u \in \mathbb{R}^{n}$ such that $u \cdot w^{\prime} \geq 0$ for all $w^{\prime} \in \mathscr{C}$ is strongly convex. Moreover, for any nonzero $u \in \mathscr{C}^{*}$ and any $w^{\prime} \in \mathscr{C}$, we have that $u \cdot w^{\prime}>0$.

We are now ready to define the space of formal Nilsson series solutions of $H_{A}(\beta)$ associated to a weight vector $w$. Note that the name Nilsson class is usually reserved for multivalued functions that satisfy tempered growth conditions (see Chapter 6.4 in [5]). In this article, we work with formal Nilsson series, except when otherwise noted.

Definition 2.6. Let $w$ be a weight vector for the hypergeometric system $H_{A}(\beta)$. Write $\log (x)=\left(\log \left(x_{1}\right), \ldots, \log \left(x_{n}\right)\right)$. A formal solution $\phi$ of $H_{A}(\beta)$ is called a basic Nilsson solution of $H_{A}(\beta)$ in the direction of $w$ if it has the form

$$
\phi=\sum_{u \in C} x^{v+u} p_{u}(\log (x)),
$$

for some vector $v \in \mathbb{C}^{n}$, and it satisfies 
1) $C$ is contained in $\mathscr{C}^{*} \cap \operatorname{ker}_{\mathbb{Z}}(A)$, where $\mathscr{C}$ is an open cone containing $w$ as in Definition 2.4;

2) the $p_{u}$ are polynomials, and there exists $K \in \mathbb{Z} \operatorname{such}$ that $\operatorname{deg}\left(p_{u}\right) \leq K$ for all $u \in C$;

3) $p_{0} \neq 0$.

The set $\operatorname{supp}(\phi)=\left\{u \in C \mid p_{u} \neq 0\right\} \subset \operatorname{ker}_{\mathbb{Z}}(A)$ is called the support of $\phi$.

The $\mathbb{C}$-span of the basic Nilsson solutions of $H_{A}(\beta)$ in the direction of $w$ is called the space of formal Nilsson series solutions of $H_{A}(\beta)$ in the direction of $w$ and is denoted by $\mathcal{N}_{w}\left(H_{A}(\beta)\right)$.

In what follows, we make a detailed study of $\mathcal{N}_{w}\left(H_{A}(\beta)\right)$. One of our main results, Theorem 6.4 , states that, for fixed $A$ and $\beta$, there exists a weight vector $w$ such that $\operatorname{dim}\left(\mathcal{N}_{w}\left(H_{A}(\beta)\right)\right)=\operatorname{rank}\left(H_{A}(\beta)\right)$.

Let $w$ be a weight vector for $H_{A}(\beta)$. If $\phi=\sum_{a, b} c_{a b} x^{a} \log (x)^{b}$ is a non zero element of $\mathcal{N}_{w}\left(H_{A}(\beta)\right)$ then, by Proposition 2.5.2 in [24], the set of real parts $\left\{\operatorname{Re}(a \cdot w) \mid c_{a b} \neq 0\right.$ for some $\left.b\right\}$ achieves a (finite) minimum, denoted by $\mu(\phi)$, and the subseries of $\phi$ whose terms are $c_{a b} x^{a} \log (x)^{b}$ such that $c_{a b} \neq 0$ and $\operatorname{Re}(a \cdot w)=$ $\mu(\phi)$ is finite. We call this finite sum the initial series of $\phi$ with respect to $w$ and we denote it by $\operatorname{in}_{w}(\phi)$. The reason for including the third condition in Definition 2.6 is to ensure that $\operatorname{in}_{w}(\phi)=x^{v} p_{0}(\log (x))$. Non-basic Nilsson solutions of $H_{A}(\beta)$ will play a role in Section 5 .

Remark 2.7. Given a weight vector $w$, we may replace the first requirement in Definition 2.6 by either of the following equivalent conditions:

i) $C \subset \operatorname{ker}_{\mathbb{Z}}(A)$ and there exists an open neighborhood $U$ of $w$ such that, for all $w^{\prime} \in U$ and all $u \in C \backslash\{0\}$, we have $w^{\prime} \cdot u>0$.

ii) There exist $\mathbb{R}$-linearly independent $\gamma_{1}, \ldots, \gamma_{n} \in \mathbb{Q}^{n}$ with $w \cdot \gamma_{i}>0$ for all $i=1, \ldots, n$, such that $C \subset\left(\mathbb{R}_{\geq 0} \gamma_{1}+\cdots+\mathbb{R}_{\geq 0} \gamma_{n}\right) \cap \operatorname{ker}_{\mathbb{Z}}(A)$.

To see that (i) is equivalent to the first condition in Definition 2.6, note first that, if the latter is true, we may use $U=\mathscr{C}$. The proof that (i) and (ii) are equivalent is straightforward. If (ii) holds, we may take $\mathscr{C}$ equal to the interior of the cone $\mathbb{R}_{\geq 0} \gamma_{1}+\cdots+\mathbb{R}_{\geq 0} \gamma_{n}$.

The following lemma allows us to manipulate formal Nilsson solutions of $H_{A}(\beta)$ in the direction of a weight vector.

Lemma 2.8. Let $\phi_{1}, \ldots, \phi_{k} \in \mathcal{N}_{w}\left(H_{A}(\beta)\right)$.

1) If the initial series $\operatorname{in}_{w}\left(\phi_{1}\right), \ldots, \operatorname{in}_{w}\left(\phi_{k}\right)$ are $\mathbb{C}$-linearly independent, then so are the series $\phi_{1}, \ldots, \phi_{k}$.

2) If $\phi_{1}, \ldots, \phi_{k}$ are $\mathbb{C}$-linearly independent, there exists a $k \times k$ complex matrix $\left(\lambda_{i j}\right)$ such that the initial series of $\psi_{i}=\sum_{j=1}^{k} \lambda_{i j} \phi_{j}$ for $i=1, \ldots, k$ are $\mathbb{C}$-linearly independent.

Proof. Combine Theorem 2.5.5, Lemma 2.5.6(2), and Proposition 2.5.7 from [24], which hold for formal Nilsson series. 
Definition 2.9. A vector $v \in \mathbb{C}$ is an exponent of $H_{A}(\beta)$ with respect to a weight vector $w$ if $x^{v}$ is a solution of $\operatorname{in}_{(-w, w)}\left(H_{A}(\beta)\right)$.

Note that if $w$ is a weight vector for $H_{A}(\beta)$ and $\mathscr{C}$ is a strongly convex open cone as in Definition 2.4, then for any $w^{\prime} \in \mathscr{C}$, the exponents of $H_{A}(\beta)$ with respect to $w$ and $w^{\prime}$ coincide, because $\operatorname{in}_{(-w, w)}\left(H_{A}(\beta)\right)=\operatorname{in}_{\left(-w^{\prime}, w^{\prime}\right)}\left(H_{A}(\beta)\right)$. Moreover, the basic Nilsson solutions of $H_{A}(\beta)$ in the direction of $w$ and $w^{\prime}$ are the same, and therefore $\mathcal{N}_{w}\left(H_{A}(\beta)\right)=\mathscr{N}_{w^{\prime}}\left(H_{A}(\beta)\right)$.

Let $w$ be a weight vector for $H_{A}(\beta)$. Since $H_{A}(\beta)$ is a holonomic ideal for all $\beta$ (see Theorem 3.9 in [1]), the ideal $\operatorname{in}_{(-w, w)}\left(H_{A}(\beta)\right)$ is holonomic as well, and its holonomic rank is at most $\operatorname{rank}\left(H_{A}(\beta)\right)$, by Theorem $2.1 \mathrm{in}$ [24]. This implies that the set of exponents of $H_{A}(\beta)$ with respect to $w$ is finite, since the monomial functions corresponding to different exponents are linearly independent.

Lemma 2.10. If $\phi$ is a basic Nilsson solution of $H_{A}(\beta)$ in the direction of $w$ as in (2.1), then $v$ is an exponent of $H_{A}(\beta)$ with respect to $w$.

Proof. Since $w$ is a weight vector, $w \cdot u>0$ for all nonzero $u \in \mathscr{C}^{*}$. As $p_{0} \neq 0$, we have that $\operatorname{in}_{w}(\phi)=x^{v} p_{0}(\log (x))$, and therefore that $x^{v} p_{0}(\log (x))$ is a solution of $\operatorname{in}_{(-w, w)}\left(H_{A}(\beta)\right)$ by Theorem 2.5.5 in [24]. But then $x^{v}$ is a solution of $\operatorname{in}_{(-w, w)}\left(H_{A}(\beta)\right)$ by Theorems 2.3.3(2) and 2.3.11 in [24].

We compare the dimension of the space of formal Nilsson solutions of $H_{A}(\beta)$ in the direction of $w$ with the holonomic rank of the associated initial ideal.

Proposition 2.11. Let $w$ be a weight vector for $H_{A}(\beta)$. Then

$$
\operatorname{dim}_{\mathbb{C}}\left(\mathcal{N}_{w}\left(H_{A}(\beta)\right)\right) \leq \operatorname{rank}\left(\operatorname{in}_{(-w, w)}\left(H_{A}(\beta)\right)\right) .
$$

Proof. Choose $\psi_{1}, \ldots, \psi_{k}$ linearly independent elements of $\mathcal{N}_{w}\left(H_{A}(\beta)\right)$. The second part of Lemma 2.8 allows us to assume that $\operatorname{in}_{w}\left(\psi_{1}\right), \ldots, \operatorname{in}_{w}\left(\psi_{k}\right)$ are linearly independent solutions of the initial system $\operatorname{in}_{(-w, w)}\left(H_{A}(\beta)\right)$. These initial series have a non empty common open domain of convergence since they have a finite number of terms. Therefore $\operatorname{dim}_{\mathbb{C}}\left(\mathcal{N}_{w}\left(H_{A}(\beta)\right)\right)$ cannot exceed the holonomic rank of $\operatorname{in}_{(-w, w)}\left(H_{A}(\beta)\right)$.

We show in Corollary 4.12 that this inequality is, in fact, an equality for generic $\beta$. If $I_{A}$ is a homogeneous ideal, then Theorems 2.4.9, 2.5.1, and 2.5.16 in [24] imply that $\operatorname{dim}_{\mathbb{C}} \mathcal{N}_{w}\left(H_{A}(\beta)\right), \operatorname{rank}\left(\operatorname{in}_{(-w, w)}\left(H_{A}(\beta)\right)\right)$, and $\operatorname{rank}\left(H_{A}(\beta)\right)$ are the same. However, if $I_{A}$ is not homogeneous, $\operatorname{rank}\left(\operatorname{in}_{(-w, w)}\left(H_{A}(\beta)\right)\right)$ does not always equal $\operatorname{rank}\left(H_{A}(\beta)\right)$ (see Corollary 4.13).

\section{Homogenization of formal Nilsson solutions of $H_{A}(\beta)$}

The goal of this section is to obtain the solutions of the system $H_{A}(\beta)$ by solving a related hypergeometric system that is regular holonomic. For generic parameters, this idea was used in other works, such as [22]; here, we require no genericity hypotheses on $\beta$. The key concept is that of homogenization. 
Notation 3.1. Throughout this article, the letter $\rho$ is used to indicate the homogenization of various objects: polynomials, ideals, and later on, Nilsson series.

If $f \in \mathbb{C}\left[\partial_{1}, \ldots, \partial_{n}\right]$ is a polynomial, we denote by $\rho(f) \in \mathbb{C}\left[\partial_{0}, \partial_{1}, \ldots, \partial_{n}\right]$ its homogenization, that is,

$$
f=\sum_{u \in \mathbb{N}^{n}} c_{u} \partial^{u} \Longrightarrow \rho(f)=\sum_{u \in \mathbb{N}^{n}} c_{u} \partial_{0}^{\operatorname{deg}(f)-|u|} \partial^{u}, \quad|u|=u_{1}+\cdots+u_{n} .
$$

If $I \subseteq \mathbb{C}\left[\partial_{1}, \ldots, \partial_{n}\right]$ is an ideal, then $\rho(I) \subseteq \mathbb{C}\left[\partial_{0}, \partial_{1}, \ldots, \partial_{n}\right]$ denotes the ideal generated by the homogenizations $\rho(f)$ for all $f \in I$.

If $A=\left[a_{i j}\right]$ is a $d \times n$ integer matrix, then $\rho(A) \in \mathbb{Z}^{(d+1) \times(n+1)}$ is obtained by attaching a column of zeros to the left of $A$, and then attaching a row of ones to the resulting matrix, namely

$$
\rho(A)=\left[\begin{array}{cccc}
1 & 1 & \ldots & 1 \\
0 & a_{11} & \ldots & a_{1 n} \\
\vdots & \vdots & \ddots & \vdots \\
0 & a_{d 1} & \ldots & a_{d n}
\end{array}\right] .
$$

Note that $\rho\left(I_{A}\right)=I_{\rho(A)}$.

Let $w$ be a weight vector for $H_{A}(\beta)$ and let $\mathscr{C}$ be a cone as in Definition 2.4. In particular $w \in \mathbb{R}_{>0}^{n}$. For fixed $\beta_{0} \in \mathbb{C}$, consider the (regular holonomic) hypergeometric system associated to the matrix $\rho(A)$ from Notation 3.1 and the vector $\left(\beta_{0}, \beta\right) \in \mathbb{C}^{n+1}$, which is denoted by $H_{\rho(A)}\left(\beta_{0}, \beta\right)$.

Remark 3.2. Since $(1, \ldots, 1)$ belongs to the rational rowspan of $\rho(A)$, weight vectors for $H_{\rho(A)}\left(\beta_{0}, \beta\right)$ are not required to have positive coordinates (see Remark 2.5). We wish to use $(0, w)$ as a weight vector for $H_{\rho(A)}\left(\beta_{0}, \beta\right)$, but this vector may not satisfy the definition. This can be remedied by perturbing $w$ as follows. The set of weight vectors for $H_{\rho(A)}\left(\beta_{0}, \beta\right)$ is an open dense subset of $\mathbb{R}^{n+1}$. Therefore, given $w$ (and $\mathscr{C}$ ) as before, there exists $\alpha=\left(\alpha_{0}, \ldots, \alpha_{n}\right) \in \mathbb{R}^{n+1}$ and $\varepsilon_{0}>0$ such that $(0, w)+\varepsilon \alpha$ is a weight vector for $H_{\rho(A)}\left(\beta_{0}, \beta\right)$ for all $0<\varepsilon<\varepsilon_{0}$. But then the argument in Remark 2.5 shows that $(0, w)+\varepsilon \alpha-\varepsilon \alpha_{0}(1, \ldots, 1)$ is also a weight vector for $H_{\rho(A)}\left(\beta_{0}, \beta\right)$. If $\varepsilon$ is sufficiently small, then $w^{\prime}=w+\varepsilon\left(\left(\alpha_{1}, \ldots, \alpha_{n}\right)-\alpha_{0}(1, \ldots, 1)\right)$ belongs to an open cone $\mathscr{C}$ as in Definition 2.4. This means that we can use $w^{\prime}$ instead of $w$ as weight vector for $H_{A}(\beta)$, with the same open cone, initial ideals, and basic Nilsson solutions as $w$, and guarantee that $\left(0, w^{\prime}\right)$ is a weight vector for $H_{\rho(A)}\left(\beta_{0}, \beta\right)$. The previous argument justifies assuming, as we do from now on, that any time we choose a weight vector $w$ for $H_{A}(\beta)$, the vector $(0, w)$ is a weight vector for $H_{\rho(A)}\left(\beta_{0}, \beta\right)$.

We choose a weight vector $w$, and we wish to use the regular system $H_{\rho(A)}\left(\beta_{0}, \beta\right)$ to study the solutions of $H_{A}(\beta)$. The matrix $\rho(A)$ is fixed, but we have freedom in the choice of the parameter $\beta_{0} \in \mathbb{C}$, and it is convenient to assume that $\beta_{0}$ is generic. The correct notion of genericity for $\beta_{0}$ can be found in Definition 3.10. Under that hypothesis, our objective is to construct an injective linear map

$$
\rho: \mathcal{N}_{w}\left(H_{A}(\beta)\right) \longrightarrow \mathscr{N}_{(0, w)}\left(H_{\rho(A)}\left(\beta_{0}, \beta\right)\right)
$$


whose image is described in Theorem 3.18. Since by definition $\mathcal{N}_{w}\left(H_{A}(\beta)\right)$ has a basis consisting of basic Nilsson solutions of $H_{A}(\beta)$ in the direction of $w$, it is enough to define our map on those series, and check that their images are linearly independent. For some weight vectors, $\rho$ is guaranteed to be surjective (Proposition 7.1). However, if the cone over the columns of $A$ is strongly convex and $I_{A}$ is not homogeneous, there always exist weights for which surjectivity fails (Proposition 7.4).

Let $\phi=\sum_{u \in C} x^{v+u} p_{u}(\log (x))$ be a basic Nilsson solution of $H_{A}(\beta)$ in the direction of $w$ as in (2.1). Since $\phi$ is annihilated by the Euler operators $E_{1}-\beta_{1}, \ldots$, $E_{d}-\beta_{d}$, the polynomials $p_{u}$ appearing in $\phi$ belong to the symmetric algebra of the lattice $\operatorname{ker}_{\mathbb{Z}}(A)$ by Proposition 5.2 in [23].

If $v$ is a vector, denote by $|v|$ the sum of its coordinates. Then, for any $\gamma \in$ $\operatorname{ker}_{\mathbb{Z}}(A),(-|\gamma|, \gamma) \in \operatorname{ker}_{\mathbb{Z}}(\rho(A))$. This inclusion $\operatorname{ker}_{\mathbb{Z}}(A) \hookrightarrow \operatorname{ker}_{\mathbb{Z}}(\rho(A))$ induces an injection (denoted by $\hat{\cdot}$ ) between the corresponding symmetric algebras of the lattices of $\operatorname{ker}_{\mathbb{Z}}(A)$ and $\operatorname{ker}_{\mathbb{Z}}(\rho(A))$. In concrete terms, let $\left\{\gamma_{1}, \ldots, \gamma_{n-d}\right\} \subset \mathbb{Z}^{n}$ be a $\mathbb{Z}$-basis of $\operatorname{ker}_{\mathbb{Z}}(A)$. We can write an element $p$ of the symmetric algebra of $\operatorname{ker}_{\mathbb{Z}}(A)$ as follows:

$$
p\left(t_{1}, \ldots, t_{n}\right)=\sum_{\alpha \in \mathbb{N}^{n-d}} c_{\alpha} \prod_{j=1}^{n-d}\left(\gamma_{j} \cdot\left(t_{1}, \ldots, t_{n}\right)\right)^{\alpha_{j}}
$$

and then we have

$$
\hat{p}\left(t_{0}, t_{1}, \ldots, t_{n}\right)=\sum_{\alpha \in \mathbb{N}^{n-d}} c_{\alpha} \prod_{j=1}^{n-d}\left(\left(-\left|\gamma_{j}\right|\right) t_{0}+\gamma_{j} \cdot\left(t_{1}, \ldots, t_{n}\right)\right)^{\alpha_{j}} .
$$

Note that $\hat{p}\left(\log \left(x_{0}\right), \ldots, \log \left(x_{n}\right)\right)$ specializes to $p(\log (x))$ when $x_{0}=1$, or equivalently, when $\log \left(x_{0}\right)=0$.

The formal definition of the homogenization of a basic Nilsson solution

$$
\phi=\sum_{u \in C} x^{v+u} p_{u}(\log (x))
$$

of $H_{A}(\beta)$ in the direction of $w$ is:

$$
\rho(\phi)=\sum_{u \in C} \partial_{0}^{|u|} x_{0}^{\beta_{0}-|v|} x^{v+u} \widehat{p_{u}}\left(\log \left(x_{0}\right), \ldots, \log \left(x_{n}\right)\right) .
$$

If $|u| \geq 0$ for all $u \in C$, the above formula makes sense, and it easily checked that $\rho(\phi)$ is a basic Nilsson solution of $H_{\rho(A)}\left(\beta_{0}, \beta\right)$ in the direction of $(0, w)$. The bulk of the work in this section concerns the definition and properties of the operator $\partial_{0}^{k}$ when $k \in \mathbb{Z}_{<0}$.

We point out that there is one case when the elements of the supports of all basic Nilsson solutions of $H_{A}(\beta)$ in the direction of $w$ are guaranteed to have nonnegative coordinate sum, namely when the weight vector $w$ is close to $(1, \ldots, 1)$. We make this notion precise in the following definition. 
Definition 3.3. Let $w$ be weight vector for $H_{A}(\beta)$. We say that $w$ is a perturbation of $w_{0} \in \mathbb{R}_{>0}^{n}$ if there exists an open cone $\mathscr{C}$ as in Definition 2.4 with $w \in \mathscr{C}$, such that $w_{0}$ lies in the closure of $\mathscr{C}$.

Suppose that $\phi=x^{v} \sum_{u \in C} x^{u} p_{u}(\log (x))$ is a basic Nilsson solution of $H_{A}(\beta)$ in the direction of a weight vector $w$ which is a perturbation of $(1, \ldots, 1)$. Since $u \in C$ implies $u \in \mathscr{C}^{*}=(\overline{\mathscr{C}})^{*}$, we have $u \cdot w \geq 0$ for all $u \in C$. But then, as $w$ is a perturbation of $(1, \ldots, 1)$, it follows that $|u|=u \cdot(1, \ldots, 1) \geq 0$ for all $u \in C$. Also, $C \subseteq \mathbb{Z}^{n}$ implies $|u| \in \mathbb{Z}$ for $u \in C$. Therefore, the operator $\partial_{0}^{|u|}$ is defined, and so is (3.3).

As we mentioned before, in order to work with other weight vectors, we must define the operator $\partial_{0}^{k}$ when $k$ is negative. We need to introduce key combinatorial objects from [27], the standard pairs of a monomial ideal $I$, which correspond to the associated primes of $I$ (see [27]).

Definition 3.4. Let $I \subseteq \mathbb{C}\left[\partial_{1}, \ldots, \partial_{n}\right]$ be a monomial ideal. Consider the variety

$$
\mathscr{V}={\overline{\left\{u \in \mathbb{N}^{n} \subset \mathbb{C}^{n} \mid x^{u} \notin I\right\}}}^{\text {Zariski }}
$$

whose irreducible components are integer translates of coordinate spaces. For $\sigma \subseteq\{1, \ldots, n\}$, denote $\mathbb{C}^{\sigma}=\left\{\left(t_{1}, \ldots, t_{n}\right) \mid t_{i}=0\right.$ for $\left.i \notin \sigma\right\}$. A pair $\left(\partial^{\alpha}, \sigma\right)$, where $\alpha \in \mathbb{N}^{n}$ and $\sigma \subseteq\{1, \ldots, n\}$, is called a standard pair of $I$ if $\alpha+\mathbb{C}^{\sigma}$ is an irreducible component of $\mathscr{V}$ and $\alpha$ is the coordinatewise minimal element of $\left(\alpha+\mathbb{C}^{\sigma}\right) \cap \mathbb{N}^{n}$.

Note that, if $\left(\partial^{\alpha}, \sigma\right)$ is a standard pair of a monomial ideal $I \subseteq \mathbb{C}[\partial]$, then $\alpha_{i}=0$ for all $i \notin \sigma$. A standard pair is top-dimensional if the corresponding associated prime is minimal, and it is embedded otherwise. We denote by $\mathscr{T}(I)$ the set of top-dimensional standard pairs of $I$, and define top $(I)$ to be the intersection

$$
\operatorname{top}(I)=\bigcap_{\left(\partial^{\alpha}, \sigma\right) \in \mathscr{T}(I)}\left\langle\partial_{i}^{\alpha_{i}+1} \mid i \notin \sigma\right\rangle .
$$

Definition 3.5. A vector $v \in \mathbb{C}^{n}$ is a fake exponent of $H_{A}(\beta)$ with respect to $w$ if the function $x^{v}$ is a solution of the left $D$-ideal $\operatorname{in}_{w}\left(I_{A}\right)+\langle E-\beta\rangle$.

Note that, as $\operatorname{in}_{w}\left(I_{A}\right)+\langle E-\beta\rangle \subseteq \operatorname{in}_{(-w, w)}\left(H_{A}(\beta)\right)$, all exponents of $H_{A}(\beta)$ with respect to $w$ (Definition 2.9) are also fake exponents. In general, this containment is strict, but fake exponents are easier to study combinatorially than (true) exponents. This is illustrated by the following result, a consequence of Lemma 4.1.3 in [24], which links fake exponents and standard pairs.

Lemma 3.6. Let $w$ be a weight vector for $H_{A}(\beta)$ and let $\left(\partial^{\alpha}, \sigma\right)$ be a standard pair of $\operatorname{in}_{w}\left(I_{A}\right)$. If there exists $v \in \mathbb{C}^{n}$ such that $A \cdot v=\beta$ and $v_{i}=\alpha_{i}$ for all $i \notin \sigma$, then $v$ is a fake exponent of $H_{A}(\beta)$ with respect to $w$. All fake exponents arise this way.

We also need to consider standard pairs of initial ideals of $I_{\rho(A)}$. Recall that the initial ideal $\operatorname{in}_{(0, w)}\left(I_{\rho(A)}\right)$ is a monomial ideal, as $(0, w)$ is a weight vector for $H_{\rho(A)}\left(\beta_{0}, \beta\right)$. 
Definition 3.7. A standard pair of $\operatorname{in}_{(0, w)}\left(I_{\rho(A)}\right)$ is said to pass through zero if $0 \in \sigma$.

Remark 3.8. If a fake exponent $v$ of $H_{A}(\beta)$ exists for a standard pair $\left(\partial^{\alpha}, \sigma\right)$ of $\operatorname{in}_{w}\left(I_{A}\right)$, it is unique by Corollary 3.2.9 in [24]; this justifies using the notation $v=\beta^{\left(\partial^{\alpha}, \sigma\right)}$.

Definition 3.9. We say that a basic Nilsson solution of $H_{A}(\beta)$ as in (2.1) is associated to the standard pair $\left(\partial^{\alpha}, \sigma\right)$ if $v$ is the (fake) exponent corresponding to this standard pair.

The following definition gives the correct notion of genericity for $\beta_{0} \in \mathbb{C}$ so that we can study the solutions of $H_{A}(\beta)$ using those of $H_{\rho(A)}\left(\beta_{0}, \beta\right)$.

Definition 3.10. Let $w$ be a weight vector for $H_{A}(\beta)$. We say that $\beta_{0} \in \mathbb{C}$ is a homogenizing value for $A, \beta$, and $w$ if $\beta_{0} \notin \mathbb{Z}$ and for any fake exponent $v$ of $H_{A}(\beta)$ with respect to $w$, the quantity $v_{0}$ defined by

$$
v_{0}:=\beta_{0}-\sum_{j=1}^{n} v_{j}
$$

is not an integer number.

Given a weight vector $w$ for $H_{A}(\beta)$, we fix a homogenizing value $\beta_{0}$ for $A, \beta$, and $w$. Let $\phi$ as in (2.1) be a basic Nilsson solution of $H_{A}(\beta)$ in the direction of $w$. We want to construct a basic Nilsson solution $\rho(\phi)$ of $H_{\rho(A)}\left(\beta_{0}, \beta\right)$ in the direction of $(0, w)$. The following lemma tells us how to differentiate logarithmic terms.

Lemma 3.11 (Lemma 5.3 in [23]). Let $h$ be a polynomial in $n$ variables, $\nu \in \mathbb{N}^{n}$ and $s \in \mathbb{C}^{n}$. Then

$$
\partial^{\nu} x^{s} h(\log (x))=x^{s-\nu}\left(\sum_{0 \leq \nu^{\prime} \leq \nu} \lambda_{\nu^{\prime}}\left[\partial^{\nu-\nu^{\prime}} h\right](\log (x))\right)
$$

where the sum is over nonnegative integer vectors $\nu^{\prime}$ that are coordinatewise smaller than $\nu$, and the $\lambda_{\nu^{\prime}}$ are certain complex numbers.

The following result allows us to define $\partial_{0}^{k}$ when $k$ is a negative integer.

Lemma 3.12. Let $\hat{p}$ be a polynomial in $n+1$ variables, and $s \in \mathbb{C}^{n}$. If $s_{0} \in \mathbb{C}$ and $s_{0} \neq-1$, there exists a unique polynomial $\hat{q}$ with $\operatorname{deg}(\hat{q})=\operatorname{deg}(\hat{p})$ such that

$$
\partial_{0}\left[x_{0}^{s_{0}+1} x^{s} \hat{q}\left(\log \left(x_{0}\right), \ldots, \log \left(x_{n}\right)\right)\right]=x_{0}^{s_{0}} x^{s} \hat{p}\left(\log \left(x_{0}\right), \ldots, \log \left(x_{n}\right)\right) .
$$

Proof. Writing

$$
\hat{p}(\log (x))=\sum_{i=0}^{k} p_{i}\left(\log \left(x_{1}\right), \ldots, \log \left(x_{n}\right)\right) \log \left(x_{0}\right)^{i}
$$


and

$$
\hat{q}(\log (x))=\sum_{i=0}^{k} q_{i}\left(\log \left(x_{1}\right), \ldots, \log \left(x_{n}\right)\right) \log \left(x_{0}\right)^{i},
$$

we can equate coefficients in (3.4) to obtain

$$
p_{k}=\left(s_{0}+1\right) q_{k} ; \quad p_{i}=\left(s_{0}+1\right) q_{i}+(i+1) q_{i+1}, \quad 0 \leq i \leq k-1 .
$$

Therefore

$$
q_{i}=\sum_{\ell=0}^{k-i}(-1)^{\ell} \frac{\prod_{j=1}^{\ell}(i+j)}{\left(s_{0}+1\right)^{\ell}} p_{i+\ell}, \quad 0 \leq \ell \leq k,
$$

where the empty product is defined to be 1.

Definition 3.13. With the notation of Lemma 3.12, define

$$
\partial_{0}^{-1}\left[x_{0}^{s_{0}} x^{s} \hat{p}\left(\log \left(x_{0}\right), \ldots, \log \left(x_{n}\right)\right)\right]=x_{0}^{s_{0}+1} x^{s} \hat{q}\left(\log \left(x_{0}\right), \ldots, \log \left(x_{n}\right)\right) .
$$

Note that if $s_{0} \neq-2, \ldots,-k$, the construction of $\partial_{0}^{-1}$ can be iterated $(k-1)$ times. We denote by $\partial_{0}^{-k}\left[x_{0}^{s_{0}} x^{s} \hat{p}\left(\log \left(x_{0}\right), \ldots, \log \left(x_{n}\right)\right)\right]$ the outcome of this procedure.

Lemma 3.14. Use the same notation and hypotheses as in Lemma 3.12, and assume furthermore that $x_{0}^{s_{0}} x^{s} \hat{p}(\log (x))$ is a solution of $\left\langle E_{0}-\beta_{0}, E-\beta\right\rangle$. Then $\partial_{0}^{-1}\left[x_{0}^{s_{0}} x^{s} \hat{p}(\log (x))\right]$ is a solution of $\left\langle E_{0}-\left(\beta_{0}+1\right), E-\beta\right\rangle$. If $s_{0} \neq-2, \ldots,-k$, then $\partial_{0}^{-k}\left[x_{0}^{s_{0}} x^{s} \hat{p}(\log (x))\right]$ is a solution of the system $\left\langle E_{0}-\left(\beta_{0}+k\right), E-\beta\right\rangle$.

Proof. If $i>0, \partial_{0}\left(E_{i}-\beta_{i}\right)=\left(E_{i}-\beta_{i}\right) \partial_{0}$, so that

$$
\begin{aligned}
\partial_{0}\left(E_{i}-\beta_{i}\right)\left(\partial_{0}^{-1} x_{0}^{s_{0}} x^{s} \hat{p}(\log (x))\right) & =\left(E_{i}-\beta_{i}\right) \partial_{0}\left(\partial_{0}^{-1} x_{0}^{s_{0}} x^{s} \hat{p}(\log (x))\right) \\
& =\left(E_{i}-\beta_{i}\right) x_{0}^{s_{0}} x^{s} \hat{p}(\log (x))=0 .
\end{aligned}
$$

This means that $\left(E_{i}-\beta_{i}\right)\left(\partial_{0}^{-1} x_{0}^{s_{0}} x^{s} \hat{p}(\log (x))\right)$ is constant with respect to $x_{0}$. On the other hand, $s_{0}+1 \neq 0$ and $\left(E_{i}-\beta_{i}\right)\left(\partial_{0}^{-1} x_{0}^{s_{0}} x^{s} \hat{p}(\log (x))\right)$ is a multiple of $x^{s_{0}+1}$. Thus, in order to be constant with respect to $x_{0},\left(E_{i}-\beta_{i}\right)\left(\partial_{0}^{-1} x_{0}^{s_{0}} x^{s} \hat{p}(\log (x))\right)$ has to vanish. For $i=0$, the argument is similar since $\partial_{0}\left(E_{0}-\left(\beta_{0}+1\right)\right)=\left(E_{0}-\beta_{0}\right) \partial_{0}$. The last assertion follows by induction on $k$.

Lemma 3.15. Let $\nu \in \mathbb{N}^{n}, k \in \mathbb{Z}$, and assume that $s_{0} \notin \mathbb{Z}$. Then

$$
\partial^{\nu}\left[\partial_{0}^{k}\left[x_{0}^{s_{0}} x^{s} \hat{p}\left(\log \left(x_{0}\right), \ldots, \log \left(x_{n}\right)\right)\right]\right]=\partial_{0}^{k}\left[\partial^{\nu}\left[x_{0}^{s_{0}} x^{s} \hat{p}\left(\log \left(x_{0}\right), \ldots, \log \left(x_{n}\right)\right)\right]\right] .
$$

Proof. This is clear if $k \geq 0$. For $k<0$, the result follows by induction from the uniqueness part of Lemma 3.12.

We are now ready to define the homogenization of a basic Nilsson solution of $H_{A}(\beta)$. 
Definition 3.16. Let $\phi=x^{v} \sum_{u \in C} x^{u} p_{u}(\log (x))$ be a basic Nilsson solution of $H_{A}(\beta)$ in the direction of a weight vector $w$, and let $\beta_{0} \in \mathbb{C}$ a homogenizing value for $A, \beta$ and $w$, so that $v_{0}=\beta_{0}-\sum_{i=1}^{n} v_{i}$ is not an integer. We define

$$
\rho(\phi)=\sum_{u \in C} \partial_{0}^{|u|}\left[x_{0}^{v_{0}} x^{v+u} \widehat{p_{u}}\left(\log \left(x_{0}\right), \log \left(x_{1}\right), \ldots, \log \left(x_{n}\right)\right)\right]
$$

where $\widehat{p_{u}}$ is obtained from $p_{u}$ as in (3.2).

Note that if $\phi_{1}$ and $\phi_{2}$ are basic Nilsson solutions of $H_{A}(\beta)$ and $\lambda \in \mathbb{C}$ is such that $\phi_{1}+\lambda \phi_{2}$ is also basic, then $\rho\left(\phi_{1}+\lambda \phi_{2}\right)=\rho\left(\phi_{1}\right)+\lambda \rho\left(\phi_{2}\right)$, as $\partial_{0}^{k}$ is linear. We now verify that the homogenization of a basic Nilsson solution of $H_{A}(\beta)$ is a basic Nilsson solution of $H_{\rho(A)}\left(\beta_{0}, \beta\right)$.

Proposition 3.17. Let $w$ be a weight vector for $H_{A}(\beta)$ and let $\beta_{0}$ be a fixed homogenizing value for $A, \beta$ and $w$. For any basic Nilsson solution $\phi=x^{v} \sum x^{u} p_{u}(\log (x))$ of $H_{A}(\beta)$ in the direction of $w$, the (formal) series $\rho(\phi)$ from Definition 3.16 is a basic Nilsson solution of $H_{\rho(A)}\left(\beta_{0}, \beta\right)$ in the direction of $(0, w)$. We extend $\rho$ linearly to obtain a map

$$
\rho: \mathcal{N}_{w}\left(H_{A}(\beta)\right) \rightarrow \mathscr{N}_{(0, w)}\left(H_{\rho(A)}\left(\beta_{0}, \beta\right)\right)
$$

Proof. Recall our assumption that, if $w$ is a weight vector for $H_{A}(\beta),(0, w)$ is a weight vector for $H_{\rho(A)}\left(\beta_{0}, \beta\right)$.

We first show that the series $\rho(\phi)$ has the shape required in Definition 2.6. Conditions 2 and 3 are clearly satisfied by the construction of the polynomials $\hat{p}_{u}$ and Lemma 3.11. Thus, it is enough to verify that $\rho(\phi)$ satisfies condition (i) from Remark 2.7. The support of $\phi$ is in bijection with the support of $\rho(\phi)$ via $u \mapsto(-|u|, u)$, which sends $\operatorname{ker}_{\mathbb{Z}}(A)$ into $\operatorname{ker}_{\mathbb{Z}}(\rho(A))$. We can assume that $C$ is the intersection of $\operatorname{ker}_{\mathbb{Z}}(A)$ with the dual $\mathscr{C}^{*}$ of an open cone $\mathscr{C}$ such that its closure $\overline{\mathscr{C}}$ is a strongly convex rational polyhedral cone of maximal dimension. Let $\left\{\gamma_{1}, \ldots, \gamma_{m}\right\}$ be a Hilbert basis of $\mathscr{C}^{*} \cap \operatorname{ker}_{\mathbb{Z}}(A)=(\overline{\mathscr{C}})^{*} \cap \operatorname{ker}_{\mathbb{Z}}(A)$. Then $w^{\prime} \cdot \gamma_{i}>0$ for all $w^{\prime} \in \mathscr{C}$ and all $i=1, \ldots, m$. Let $\delta>0$ be such that, for all $\varepsilon \in \mathbb{R}_{>0}^{n+1}$ whose Euclidean distance to the origin is $\|\varepsilon\|<\delta$, and all $i=1, \ldots, m$, we have $[(0, w)+\varepsilon] \cdot\left(-\left|\gamma_{i}\right|, \gamma_{i}\right)>0$. It follows that for any nonzero $u \in C$ and any $\tilde{w}$ in the ball centered at $(0, w)$ with radius $\delta$, we have $\tilde{w} \cdot(-|u|, u)>0$, which proves our claim.

Now we prove that $\rho(\phi)$ is a formal solution of $H_{\rho(A)}\left(\beta_{0}, \beta\right)$. Since $\widehat{p_{u}}$ belongs to the symmetric algebra of $\operatorname{ker}_{\mathbb{Z}}(\rho(A))$, and

$$
\rho(A) \cdot\left(v_{0}, v+u\right)=\left(\beta_{0}-|v|+|v|+|u|, A \cdot(v+u)\right)=\left(\beta_{0}+|u|, \beta\right),
$$

the term $x_{0}^{v_{0}} x^{v+u} \widehat{p_{u}}\left(\log \left(x_{0}\right), \log \left(x_{1}\right), \ldots, \log \left(x_{n}\right)\right)$ is a solution of the system of Euler operators $\left\langle E_{0}-\left(\beta_{0}+|u|\right), E_{1}-\beta_{1}, \ldots, E_{d}-\beta_{d}\right\rangle$. By Lemma 3.14 with $\left(s_{0}, s\right)=\left(v_{0}, v+u\right)$ and $k=-|u|$, each term of $\rho(\phi)$ is therefore a solution of $\left\langle E-\left(\beta_{0}+|u|-|u|\right), E_{1}-\beta_{1}, \ldots, E_{d}-\beta_{d}\right\rangle$. 
To verify that the elements of $I_{\rho(A)}$ annihilate $\rho(\phi)$, first note that Lemma 3.11 implies that, for any $\mu \in \operatorname{ker}_{\mathbb{Z}}(A)$,

$$
\partial^{\mu_{+}} x^{v+u} p_{u}(\log (x))=\partial^{\mu_{-}} x^{v+u-\mu} p_{u-\mu}(\log (x)),
$$

because $\phi$ is a solution of $H_{A}(\beta)$.

We claim that

$$
\partial^{\mu_{+}} x_{0}^{v_{0}} x^{v+u} \widehat{p_{u}}(\log (x))=\partial^{\mu_{-}} x_{0}^{v_{0}} x^{v+u-\mu} \widehat{p_{u-\mu}}(\log (x)) .
$$

To see this, use Lemma 3.11 and the fact that, if $i>0$ and $p$ is an element of the symmetric algebra of $\operatorname{ker}_{\mathbb{Z}}(A)$, then $\partial_{i} p$ is also in the symmetric algebra of $\operatorname{ker}_{\mathbb{Z}}(A)$, and $\partial_{i} \widehat{p}=\widehat{\partial_{i} p}$.

Now, using Lemma 3.15 with $\left(s_{0}, s\right)=\left(v_{0}, v+u\right)$ and $k=|u|$, and the fact that $v_{0}=\beta_{0}-|v| \notin \mathbb{Z}$, we obtain from

$$
\partial_{0}^{|u|} \partial^{\mu_{+}} x_{0}^{v_{0}} x^{v+u} \widehat{p_{u}}(\log (x))=\partial_{0}^{|\mu|} \partial_{0}^{|u|-|\mu|} \partial^{\mu_{-}} x_{0}^{v_{0}} x^{v+u-\mu} \widehat{p_{u-\mu}}(\log (x)),
$$

that

$$
\partial^{\mu_{+}}\left[\partial_{0}^{|u|} x_{0}^{v_{0}} x^{v+u} \widehat{p_{u}}(\log (x))\right]=\partial_{0}^{|\mu|} \partial^{\mu_{-}}\left[\partial_{0}^{|u|-|\mu|} x_{0}^{v_{0}} x^{v+u-\mu} \widehat{p_{u-\mu}}(\log (x))\right] .
$$

Assuming $|\mu|>0$, we conclude

$$
\partial^{(-|\mu|, \mu)_{+}}\left[\partial_{0}^{|u|} x_{0}^{v_{0}} x^{v+u} \widehat{p_{u}}(\log (x))\right]=\partial^{(-|\mu|, \mu)_{-}}\left[\partial_{0}^{|u|-|\mu|} x_{0}^{v_{0}} x^{v+u-\mu} \widehat{p_{u-\mu}}(\log (x))\right] .
$$

The next result shows that $\rho$ is one to one. Therefore, the inverse map $\rho^{-1}$ allows us to obtain Nilsson solutions of $H_{A}(\beta)$ from Nilsson solutions of the regular holonomic system $H_{\rho(A)}\left(\beta_{0}, \beta\right)$.

Theorem 3.18. Let $w$ be a weight vector for $H_{A}(\beta)$ and $\beta_{0}$ a homogenizing value for $A, \beta$ and $w$. The linear map

$$
\rho: \mathcal{N}_{w}\left(H_{A}(\beta)\right) \rightarrow \mathscr{N}_{(0, w)}\left(H_{\rho(A)}\left(\beta_{0}, \beta\right)\right)
$$

is injective and its image is spanned by basic Nilsson solutions of $H_{\rho(A)}\left(\beta_{0}, \beta\right)$ in the direction of $(0, w)$ associated to standard pairs of $\operatorname{in}_{(0, w)}\left(I_{\rho(A)}\right)$ that pass through zero.

Proof. If $\phi=\sum_{u \in C} x^{v+u} p_{u}(\log (x))$ is a basic Nilsson solution of $H_{A}(\beta)$ in the direction of $w$, then we have $\operatorname{in}_{(0, w)}(\rho(\phi))=x_{0}^{v_{0}} x^{v} \widehat{p_{0}}\left(\log \left(x_{0}\right), \ldots, \log \left(x_{n}\right)\right)$. Choose a basis of $\mathcal{N}_{w}\left(H_{A}(\beta)\right)$ consisting of basic Nilsson series whose initial terms are linearly independent (use the second part of Lemma 2.8). Then the initial series of their images are also linearly independent, as $\hat{p}(1, \log (x))=p(\log (x))$. Now apply the first part of Lemma 2.8 to complete the proof that $\rho$ is injective.

Observe that, by construction, $\rho\left(\mathcal{N}_{w}\left(H_{A}(\beta)\right)\right)$ is contained in the span of the basic Nilsson solutions of $H_{\rho(A)}\left(\beta_{0}, \beta\right)$ corresponding to standard pairs that pass through zero, because the powers of $x_{0}$ appearing in $\rho(\phi)$ are non-integer for any basic Nilsson solution $\phi$ of $H_{A}(\beta)$. 
To show the other inclusion, let $\psi$ be a basic Nilsson solution of $H_{\rho(A)}\left(\beta_{0}, \beta\right)$ in the direction of $(0, w)$ corresponding to a standard pair that passes through zero, with starting exponent $\left(\beta_{0}-|v|, v\right)$. We wish to prove that $\psi$ can be dehomogenized. We can write

$$
\psi=x_{0}^{\beta_{0}-|v|} x^{v} \sum_{(-|u|, u) \in \operatorname{ker}_{\mathbb{Z}}(\rho(A))} x_{0}^{-|u|} x^{u} h_{u}(\log (x)),
$$

where $\beta_{0}-|v|$ is not an integer because $\beta_{0}$ is a homogenizing value for $A, \beta$ and $w$. Then we have

$$
\partial_{0}^{|u|}\left(x_{0}^{\beta_{0}-|v|-|u|} x^{v+u} h_{u}(\log (x))\right)=x_{0}^{\beta_{0}-|v|} x^{v+u} \widehat{p_{u}}(\log (x))
$$

and use this to define $\phi=\sum x^{v+u} p_{u}(\log (x)$ ) (with the same relationship between $p$ and $\widehat{p}$ as in (3.2)). We claim that $\phi$ is a basic Nilsson solution of $H_{A}(\beta)$ and $\psi=$ $\rho(\phi)$. The proof of this claim is a reversal of the arguments in Proposition 3.17.

Definition 3.19. If $\psi \in \mathscr{N}_{(0, w)}\left(H_{\rho(A)}\left(\beta_{0}, \beta\right)\right)$ and $\phi \in \mathcal{N}_{w}\left(H_{A}(\beta)\right)$ are such that $\rho(\phi)=\psi$, we call $\phi$ a dehomogenized Nilsson series, or say that $\phi$ is the dehomogenization of $\psi$.

\section{Hypergeometric Nilsson series for generic parameters}

When the parameter vector $\beta$ is sufficiently generic (see Convention 4.2), the Nilsson solutions of $H_{A}(\beta)$ are completely determined by the combinatorics of the initial ideals of $I_{A}$. The goal of this section is to study this case in detail.

In order to precisely describe the genericity condition used in this section, we need to understand the initial ideal $\operatorname{in}_{(-w, w)}\left(H_{A}(\beta)\right)$ for generic parameter vectors.

Lemma 4.1. For $\beta$ generic,

$$
\operatorname{in}_{(-w, w)}\left(H_{A}(\beta)\right)=\operatorname{in}_{w}\left(I_{A}\right)+\langle E-\beta\rangle .
$$

Therefore, all the fake exponents of $H_{A}(\beta)$ with respect to $w$ are true exponents.

Moreover, under suitable genericity conditions for $\beta$, a better description of $\operatorname{in}_{(-w, w)}\left(H_{A}(\beta)\right)$ is available, namely

$$
\operatorname{in}_{(-w, w)}\left(H_{A}(\beta)\right)=\operatorname{top}\left(\operatorname{in}_{w}\left(I_{A}\right)\right)+\langle E-\beta\rangle .
$$

Proof. This is a version of Theorems 3.1.3 and 3.2.11 in [24] for non-homogeneous toric ideals. The same proofs hold, since $I_{A}$ is always $A$-graded.

Convention 4.2. In this section, we assume that $\beta$ is generic enough that the second displayed formula in Lemma 4.1 is satisfied, so that all exponents of $H_{A}(\beta)$ with respect to $w$ come from top-dimensional standard pairs of $\operatorname{in}_{w}\left(I_{A}\right)$.

We also require that the only integer coordinates of these exponents are the ones imposed by the corresponding standard pairs. In particular, the exponents of $H_{A}(\beta)$ with respect to $w$ have no negative integer coordinates.

Finally, we ask that no two exponents differ by an integer vector. Note that these integrality conditions force us to avoid an infinite (but locally finite) collection of affine spaces. 
The following result holds without homogeneity assumptions on the matrix $A$ :

Proposition 4.3. For any $v \in\left(\mathbb{C} \backslash \mathbb{Z}_{<0}\right)^{n}$ such that $A \cdot v=\beta$, the formal series

$$
\phi_{v}=\sum_{u \in \operatorname{ker}_{\mathbb{Z}}(A)} \frac{[v]_{u_{-}}}{[u+v]_{u_{+}}} x^{u+v}
$$

where

$$
[v]_{u_{-}}=\prod_{u_{i}<0} \prod_{j=1}^{-u_{i}}\left(v_{i}-j+1\right) ; \quad[u+v]_{u_{+}}=\prod_{u_{i}>0} \prod_{j=1}^{u_{i}}\left(v_{i}+j\right)
$$

is well defined and is annihilated by the hypergeometric D-ideal $H_{A}(\beta)$.

If $v$ is a fake exponent of $H_{A}(\beta)$ corresponding to a standard pair $\left(\partial^{a}, \sigma\right)$ of $\operatorname{in}_{w}\left(I_{A}\right)$, then $\phi_{v}$ is a basic Nilsson solution of $H_{A}(\beta)$. If moreover $\beta$ is a generic parameter vector, the support of $\phi_{v}$ is the set

$$
\operatorname{supp}\left(\phi_{v}\right)=\left\{u \in \operatorname{ker}_{\mathbb{Z}}(A) \mid u_{i}+v_{i} \geq 0 \quad \forall i \notin \sigma\right\} .
$$

Thus,

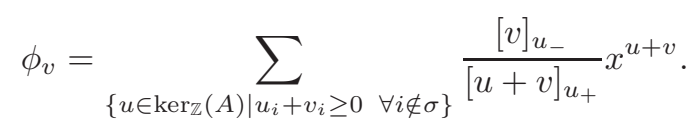

Proof. This statement is essentially a combination of Proposition 3.4.1, Theorem 3.4.2 and Lemma 3.4.6 in [24], which do not need homogeneity for $I_{A}$. It only remains to be checked that $\phi_{v}$ is a basic Nilsson solution of $H_{A}(\beta)$ in the direction of $w$. To see this, note that $u \in \operatorname{supp}\left(\phi_{v}\right)$ implies $u \cdot w \geq 0$ as is shown in the proof of Theorem 3.4.2 in [24]. Since $v$ is a fake exponent of $H_{A}(\beta)$ with respect to any $w^{\prime}$ in an open neighborhood of $w$, we have in fact that $u \cdot w>0$ for any nonzero $u$ in the support of $\phi_{v}$. This argument shows that $\phi_{v}$ satisfies the first requirement from Definition 2.6. The remaining conditions are readily verified.

\subsection{A summary of known results for homogeneous $I_{A}$ and generic $\beta$}

In this brief subsection we recall combinatorial features and convergence results for $A$-hypergeometric functions under the assumption that the toric ideal $I_{A}$ is homogeneous, which implies that $H_{A}(\beta)$ is regular holonomic. This material comes from [24].

For the purposes of this subsection only, we assume that the vector $(1, \ldots, 1)$ is contained in the rational rowspan of $A$, or equivalently, that the toric ideal $I_{A}$ is homogeneous with respect to the usual $\mathbb{Z}$-grading in $\mathbb{C}[\partial]$. We keep our weight vector $w$, and we assume the genericity on $\beta$ required in Convention 4.2. Later we apply what follows to $\rho(A),\left(\beta_{0}, \beta\right)$, and $(0, w)$.

If $v=\beta^{\left(\partial^{\alpha}, \sigma\right)} \in \mathbb{C}^{n}$ is the exponent of $H_{A}(\beta)$ associated to a (top dimensional) standard pair $\left(\partial^{\alpha}, \sigma\right)$ of $\operatorname{in}_{w}\left(I_{A}\right)$, the series $\phi_{v}=\phi_{\beta^{\left(\partial^{\alpha}, \sigma\right)}}$ converges in an open set $\mathscr{U}_{w, \varepsilon}$ of the form (6.1) for some $\varepsilon \in \mathbb{R}_{>0}^{n-d}$ by Theorem 2.5.16 in [24]. 
Let $\operatorname{conv}(A)$ be the convex hull of the columns $a_{1}, \ldots, a_{n}$ of $A$. Since the rational rowspan of $A$ contains $(1, \ldots, 1)$, there is a hyperplane $H$ off the origin such that $\operatorname{conv}(A) \subseteq H$, and as $A$ has full rank, $\operatorname{conv}(A)$ has dimension $d-1$. The normalized volume $\operatorname{vol}(A)$ is the Euclidean volume (in $H)$ of $\operatorname{conv}(A)$ normalized so that the unit simplex in the lattice $\mathbb{Z} A \cap H$ has volume one. As we have assumed that $\mathbb{Z} A=\mathbb{Z}^{d}$, the normalization is achieved by multiplying the Euclidean volume of $\operatorname{conv}(A)$ by $(d-1)$ !. We think of $A$, not just as a matrix, but as the point configuration $\left\{a_{1}, \ldots, a_{n}\right\} \subset \mathbb{Z}^{d}$. A vector $w \in \mathbb{R}_{>0}^{n}$ induces a subdivision $\Delta_{w}$ of the configuration $A$, by projecting the lower hull of $\operatorname{conv}\left(\left\{\left(w_{i}, a_{i}\right) \mid i=1, \ldots, n\right\}\right)$ onto $\operatorname{conv}(A)$ (see Chapter 8 of [26] for details). If $w$ is generic, $\Delta_{w}$ is a triangulation of $A$. Such triangulations are usually called regular, but we use the alternative term coherent. By Theorem 8.3 in [26], the radical ideal $\sqrt{\operatorname{in}_{w}\left(I_{A}\right)}$ is the Stanley-Reisner ideal of the triangulation $\Delta_{w}$, whose facet set is $\left\{\sigma \mid\left(\partial^{\alpha}, \sigma\right) \in \mathscr{T}\left(\operatorname{in}_{w}\left(I_{A}\right)\right)\right.$ for some $\left.\alpha\right\}$. We always write triangulations of $A$ as simplicial complexes on $\{1, \ldots, n\}$, but think of them geometrically: a simplex $\sigma$ in such a triangulation corresponds to the geometric simplex $\operatorname{conv}\left(\left\{a_{i} \mid i \in \sigma\right\}\right)$.

The set

$$
\left\{\phi_{\beta^{\left(\partial^{\alpha}, \sigma\right)}} \mid\left(\partial^{\alpha}, \sigma\right) \in \mathscr{T}\left(\operatorname{in}_{w}\left(I_{A}\right)\right)\right\}
$$

consists of $\operatorname{rank}\left(\operatorname{in}_{(-w, w)}\left(H_{A}(\beta)\right)\right)=\# \mathscr{T}\left(\operatorname{in}_{w}\left(I_{A}\right)\right)=\operatorname{deg}\left(I_{A}\right)=\operatorname{vol}(A)$ linearly independent series solutions of $H_{A}(\beta)$ (linear independence follows from Lemma 2.8) which have a common domain of convergence. In addition, the fact that $I_{A}$ is homogeneous implies that $H_{A}(\beta)$ is regular holonomic [16], and therefore by Theorem 2.5.1 in [24], we have that $\operatorname{rank}\left(\operatorname{in}_{(-w, w)}\left(H_{A}(\beta)\right)\right)=\operatorname{rank}\left(H_{A}(\beta)\right)$. Thus (4.4) is, in fact, a basis for the (multivalued) holomorphic solutions of $H_{A}(\beta)$ in an open set of the form $\mathscr{U}_{w, \varepsilon}$ defined in (6.1) below.

\subsection{The general case}

We now drop the homogeneity assumption on $I_{A}$, but keep the genericity assumption for $\beta$. In this subsection, we describe the space $\mathcal{N}_{w}\left(H_{A}(\beta)\right)$ using the homogenization map $\rho$ defined in Section 3. An explicit basis for $\mathcal{N}_{w}\left(H_{A}(\beta)\right)$ is constructed using the exponents of the ideal $H_{A}(\beta)$. Our first step is to relate the exponents of $H_{A}(\beta)$ with respect to $w$ to the exponents of $H_{\rho(A)}\left(\beta_{0}, \beta\right)$ with respect to $(0, w)$.

The following is a well known result, whose proof we include for the sake of completeness.

Lemma 4.4. Let $I \subset \mathbb{C}\left[\partial_{1}, \ldots, \partial_{n}\right]$ be an ideal and let $\rho(I) \subset \mathbb{C}\left[\partial_{0}, \partial_{1}, \ldots, \partial_{n}\right]$ be its homogenization. Let $w \in \mathbb{R}_{>0}^{n}$ be sufficiently generic so that $\operatorname{in}_{w}(I)$ and $\operatorname{in}_{(0, w)}(\rho(I))$ are both monomial ideals. Suppose that

$$
\operatorname{in}_{(0, w)}(\rho(I))=\bigcap Q_{i}
$$

is a primary decomposition of the monomial ideal $\operatorname{in}_{(0, w)}(\rho(I))$. Then

$$
\operatorname{in}_{w}(I)=\bigcap_{\partial_{0} \notin \sqrt{Q_{i}}}\left\langle f\left(1, \partial_{1}, \ldots, \partial_{n}\right) \mid f \in Q_{i}\right\rangle
$$

is a primary decomposition of the monomial ideal $\operatorname{in}_{w}(I)$. 
Proof. Let $f \in I$ such that $\operatorname{in}_{w}(f)$ is a monomial. Then $\operatorname{in}_{(0, w)}(\rho(f))=\partial_{0}^{h} \operatorname{in}_{w}(f)$ for some $h \in \mathbb{N}$, with $h=0$ if $f$ is homogeneous. Therefore $\operatorname{in}_{w}(I)$ is obtained by setting $\partial_{0} \mapsto 1$ in the generators of $\operatorname{in}_{(0, w)}(\rho(I))$. Now the result follows by observing that if $Q$ is primary monomial ideal one of whose generators is divisible by $\partial_{0}$, then $Q$ must contain a power of $\partial_{0}$ as a minimal generator.

Notation 4.5. Given $\beta_{0} \in \mathbb{C}$ and $v \in \mathbb{C}^{n}$, we set

$$
\rho_{\beta_{0}}(v)=\left(\beta_{0}-\sum_{i=1}^{n} v_{i}, v\right)=\left(\beta_{0}-|v|, v\right) .
$$

In particular, $\rho_{0}=\rho$ maps $\operatorname{ker}_{\mathbb{Z}}(A)$ to $\operatorname{ker}_{\mathbb{Z}}(\rho(A))$.

Lemma 4.6. Let $\beta_{0}, \beta$ be generic and le $w$ be a weight vector for $H_{A}(\beta)$. Then the map $v \mapsto \rho_{\beta_{0}}(v)$ is a bijection between the set of exponents of $H_{A}(\beta)$ with respect to $w$ and the set of exponents of $H_{\rho(A)}\left(\beta_{0}, \beta\right)$ with respect to $(0, w)$ associated to standard pairs that pass through zero.

Proof. By Lemma 4.4, $v$ is the exponent of $H_{A}(\beta)$ with respect to $w$ corresponding to a standard pair $\left(\partial^{\alpha}, \sigma\right)$, if and only if $\rho_{\beta_{0}}(v)$ is the exponent of $H_{\rho(A)}\left(\beta_{0}, \beta\right)$ with respect to $(0, w)$ corresponding to the standard pair $\left(\partial^{\alpha},\{0\} \cup \sigma\right)$.

The following result is immediate.

Lemma 4.7. Let $\beta \in \mathbb{C}^{d}$ be generic, $w$ a weight vector for $H_{A}(\beta)$ and $\beta_{0} a$ homogenizing value for $A, \beta$ and $w$. Let $v$ be an exponent of $H_{A}(\beta)$ with respect to $w$ and consider the map $\rho$ from Proposition 3.17. Then

$$
\rho\left(\phi_{v}\right)=\phi_{\left(\rho_{\beta_{0}}(v), v\right)},
$$

where $\phi_{v}, \phi_{\left(\rho_{\beta_{0}}(v), v\right)}$ are as in Proposition 4.3 .

We now come to the main result in this section.

Theorem 4.8. Let $\beta$ be generic and let $w$ be a weight vector for $H_{A}(\beta)$. Then

$$
\left\{\phi_{v} \mid v \text { is an exponent of } H_{A}(\beta) \text { with respect to } w\right\}
$$

is a basis for $\mathcal{N}_{w}\left(H_{A}(\beta)\right)$.

Proof. Fix a homogenizing value $\beta_{0}$ for $A, \beta$ and $w$, and let $\psi \in \mathcal{N}_{w}\left(H_{A}(\beta)\right)$. Then, by Theorem 3.18, $\rho(\psi) \in \mathscr{N}_{(0, w)}\left(H_{\rho(A)}\left(\beta_{0}, \beta\right)\right)$. Since $I_{\rho(A)}$ is homogeneous, $H_{\rho(A)}\left(\beta_{0}, \beta\right)$ is regular holonomic, and we can use Lemma 4.7 and the results from the previous subsection to write

$$
\rho(\psi)=\sum c_{v} \phi_{\left(\rho_{\beta_{0}}(v), v\right)}=\sum c_{v} \rho\left(\phi_{v}\right)
$$

where the sum is over the exponents of $H_{\rho(A)}\left(\beta_{0}, \beta\right)$ with respect to $(0, w)$ corresponding to standard pairs that pass through zero, and the $c_{v}$ are complex numbers. By Lemma 4.6, the sum is over the exponents of $H_{A}(\beta)$ with respect to $w$. 
But $\rho$ is injective, so (4.5) implies $\psi=\sum c_{v} \phi_{v}$. Thus $\mathcal{N}_{w}\left(H_{A}(\beta)\right)$ is contained in the $\mathbb{C}$-span of the series $\phi_{v}$ associated to the exponents of $H_{A}(\beta)$ with respect to $w$. Since the series $\phi_{v}$ are basic Nilsson solutions of $H_{A}(\beta)$ in the direction of $w$ (Proposition 4.3), the reverse inclusion follows. Linear independence is proved using Lemma 2.8.

Recall that we have assumed that, if $w$ is a weight vector for $H_{A}(\beta)$, then $(0, w)$ is a weight vector for $H_{\rho(A)}\left(\beta_{0}, \beta\right)$. In particular, this implies that the subdivision of $\rho(A)$ induced by $(0, w)$ is in fact a triangulation (see Chapter 8 of [26]).

Corollary 4.9. Assume $\beta \in \mathbb{C}^{d}$ is generic and let $w$ be a weight vector for $H_{A}(\beta)$. The dimension of the space of Nilsson solutions of $H_{A}(\beta)$ in the direction of $w$ is

$$
\operatorname{dim}_{\mathbb{C}}\left(\mathcal{N}_{w}\left(H_{A}(\beta)\right)\right)=\sum_{\substack{\sigma \text { facet of } \Delta(0, w) \\ \text { such that } 0 \in \sigma}} \operatorname{vol}(\sigma) .
$$

Proof. By Theorem 4.8, the number $\operatorname{dim}_{\mathbb{C}}\left(\mathcal{N}_{w}\left(H_{A}(\beta)\right)\right)$ is the number of exponents of $H_{A}(\beta)$ with respect to $w$, which is the number of top-dimensional standard pairs of $\operatorname{in}_{w}\left(I_{A}\right)$, because $\beta$ is generic. Using the bijection from Lemma 4.6, we conclude that $\operatorname{dim}_{\mathbb{C}}\left(\mathcal{N}_{w}\left(H_{A}(\beta)\right)\right)$ is the number of top-dimensional standard pairs of $\operatorname{in}_{(0, w)}\left(I_{\rho(A)}\right)$ that pass through zero. Given $\sigma \subset\{0,1, \ldots, n\}$ of cardinality $d+1$ such that $0 \in \sigma$, the number of top-dimensional standard pairs of $\operatorname{in}_{(0, w)}\left(I_{\rho(A)}\right)$ of the form $\left(\partial^{\alpha}, \sigma\right)$ is the multiplicity of $\left\langle\partial_{i} \mid i \notin \sigma\right\rangle$ as an associated prime of $\operatorname{in}_{(0, w)}\left(I_{\rho(A)}\right)$ by Lemma 3.3 in [27]. This number equals the normalized volume of the simplex $\{0\} \cup \sigma$ by Theorem 8.8 in [26], and the result follows.

Proposition 4.10. Suppose that $\beta$ is generic, and let $w$ be a weight vector for $H_{A}(\beta)$. Then

$$
\operatorname{rank}\left(\operatorname{in}_{(-w, w)}\left(H_{A}(\beta)\right)=\operatorname{deg}\left(\operatorname{in}_{w}\left(I_{A}\right)\right) .\right.
$$

Proof. By Lemma 4.1, in $(-w, w)\left(H_{A}(\beta)\right)=\operatorname{top}\left(\operatorname{in}_{w}\left(I_{A}\right)\right)+\langle E-\beta\rangle$. Since top $\left(\operatorname{in}_{w}\left(I_{A}\right)\right)$ is a monomial ideal, the $D$-ideals $\operatorname{in}_{(-w, w)}\left(H_{A}(\beta)\right)$ and $\left\langle x^{u} \partial^{u} \mid \partial^{u} \in \operatorname{top}\left(\operatorname{in}_{w}\left(I_{A}\right)\right)\right\rangle+$ $\langle E-\beta\rangle$ have the same holomorphic solutions.

We denote $x_{i} \partial_{i}=\theta_{i}$, and observe that $\mathbb{C}\left[\theta_{1}, \ldots, \theta_{n}\right]$ is a commutative polynomial subring of $D$. Also recall that the Euler operators $E_{i}-\beta_{i}$ belong to $\mathbb{C}[\theta]$. Since $x^{u} \partial^{u}=\prod_{i=1}^{n} \prod_{j=0}^{u_{i}-1}\left(\theta_{i}-j\right)$, Proposition 2.3.6 in [24] can be applied to conclude that the holonomic $\operatorname{rank} \operatorname{rank}\left(\operatorname{in}_{(-w, w)}\left(H_{A}(\beta)\right)\right)$ equals

$$
\operatorname{dim}_{\mathbb{C}}\left(\frac{\mathbb{C}[\theta]}{\left\langle\prod_{i=1}^{n} \prod_{j=0}^{u_{i}-1}\left(\theta_{i}-j\right) \mid \partial^{u} \in \operatorname{top}\left(\operatorname{in}_{w}\left(I_{A}\right)\right)\right\rangle+\langle E-\beta\rangle}\right) .
$$

Considered as a system of polynomial equations in $n$ variables $\left(\theta_{1}, \ldots, \theta_{n}\right)$, the zero set of the ideal $\left\langle\prod_{i=1}^{n} \prod_{j=0}^{u_{i}-1}\left(\theta_{i}-j\right) \mid \partial^{u} \in \operatorname{top}\left(\operatorname{in}_{w}\left(I_{A}\right)\right)\right\rangle$ is a subvariety of $\mathbb{C}^{n}$ consisting of $\operatorname{deg}\left(\operatorname{in}_{w}\left(I_{A}\right)\right)$ irreducible components, each of which is a translate of a $d$-dimensional coordinate subspace of $\mathbb{C}^{n}$. By Corollary 3.2.9 in [24], each of these components meets the zero set of $\langle E-\beta\rangle$ in exactly one point. Therefore, (4.6) equals $\operatorname{deg}\left(\operatorname{in}_{w}\left(I_{A}\right)\right)$, and the proof is complete. 
Corollary 4.11. Let $\beta$ be generic and let $w$ be a weight vector for $H_{A}(\beta)$. Then

$$
\operatorname{rank}\left(\operatorname{in}_{(-w, w)}\left(H_{A}(\beta)\right)\right)=\sum_{\begin{array}{c}
\sigma \text { facet of } \Delta(0, w) \\
\text { such that } 0 \in \sigma
\end{array}} \operatorname{vol}(\sigma) .
$$

Proof. We need to show that the sum on the right hand side of (4.7) equals $\operatorname{deg}\left(\operatorname{in}_{w}\left(I_{A}\right)\right)$. By Lemma 3.3 in [27], this degree equals the number of topdimensional standard pairs of $\operatorname{in}_{w}\left(I_{A}\right)$, which equals the number of top-dimensional standard pairs of $\operatorname{in}_{(0, w)}\left(I_{\rho(A)}\right)$ passing through zero by Lemma 4.6. As in the proof of Corollary 4.9, the number of such standard pairs is the desired sum.

Corollary 4.12. Suppose that $\beta$ is generic and $w$ is a weight vector for $H_{A}(\beta)$. Then

$$
\operatorname{dim}_{\mathbb{C}}\left(\mathcal{N}_{w}\left(H_{A}(\beta)\right)\right)=\operatorname{rank}\left(\operatorname{in}_{(-w, w)}\left(H_{A}(\beta)\right)\right) .
$$

Proof. Immediate from Corollary 4.9 and Corollary 4.11.

The following corollary states that, for certain weight vectors, the dimension of the space $\mathcal{N}_{w}\left(H_{A}(\beta)\right)$ equals $\operatorname{rank}\left(H_{A}(\beta)\right)$. However, this fails in general, as Example 4.14 shows. This means that, as expected, formal Nilsson series are not enough to understand the solutions of irregular hypergeometric systems.

When working with matrices $A$ whose columns are not assumed to lie in a hyperplane off the origin, $\operatorname{vol}(A)$ denotes the normalized volume of the convex hull of $\left\{0, a_{1}, \ldots, a_{n}\right\}$ with respect to the lattice $\mathbb{Z} A=\mathbb{Z}^{d}$.

Corollary 4.13. Suppose that $\beta$ is generic, and $w$ is a weight vector for $H_{A}(\beta)$. The equality

$$
\operatorname{dim}_{\mathbb{C}}\left(\mathcal{N}_{w}\left(H_{A}(\beta)\right)\right)=\operatorname{vol}(A)=\operatorname{rank}\left(H_{A}(\beta)\right)
$$

holds if and only if 0 belongs to every maximal simplex in the triangulation $\Delta_{(0, w)}$ of $\rho(A)$.

Proof. Note that $\operatorname{vol}(A)=\operatorname{vol}(\rho(A))$, which is the sum of the volumes of all the maximal simplices in $\Delta_{(0, w)}$. Therefore $\operatorname{dim}_{\mathbb{C}}\left(\mathcal{N}_{w}\left(H_{A}(\beta)\right)\right)=\operatorname{vol}(A)$ if and only if all maximal simplices in $\Delta_{(0, w)}$ pass through zero. Now use a result of Adolphson [1] that, for generic $\beta, \operatorname{rank}\left(H_{A}(\beta)\right)=\operatorname{vol}(A)$.

Example 4.14. Let

$$
A=\left[\begin{array}{lll}
1 & 0 & 1 \\
0 & 1 & 1
\end{array}\right], \text { so that } I_{A}=\left\langle\partial_{3}-\partial_{1} \partial_{2}\right\rangle .
$$

Then $\operatorname{vol}(\rho(A))=\operatorname{vol}(A)=2$ is the generic rank of $H_{A}(\beta)$ and of $H_{\rho(A)}\left(\beta_{0}, \beta\right)$.

If $w$ is a perturbation of $(1,1,1)$, we have $\operatorname{in}_{w}\left(I_{A}\right)=\left\langle\partial_{1} \partial_{2}\right\rangle$ and the corresponding triangulation is $\Delta_{(0, w)}=\{\{0,1,3\},\{0,2,3\}\}$. In this case,

$$
\operatorname{dim}_{\mathbb{C}}\left(\mathcal{N}_{w}\left(H_{A}(\beta)\right)\right)=\operatorname{vol}(\{0,1,3\})+\operatorname{vol}(\{0,2,3\})=2=\operatorname{rank}\left(H_{A}(\beta)\right) .
$$

On the other hand, if $w$ is a perturbation of $(1,1,3)$, then we have $\operatorname{in}_{w}\left(I_{A}\right)=\left\langle\partial_{3}\right\rangle$ and the corresponding triangulation is $\Delta_{(0, w)}=\{\{0,1,2\},\{1,2,3\}\}$. In this case,

$$
\operatorname{dim}_{\mathbb{C}}\left(\mathcal{N}_{w}\left(H_{A}(\beta)\right)\right)=\operatorname{vol}(\{0,1,2\})=1<\operatorname{rank}\left(H_{A}(\beta)\right) .
$$




\section{Logarithm-free Nilsson series}

If we assume that $\beta$ is generic, then all of the Nilsson solutions of $H_{A}(\beta)$ are automatically logarithm-free. We now turn our attention to the logarithm-free Nilsson solutions of $H_{A}(\beta)$ without any assumptions on the parameter $\beta$.

Definition 5.1. For a vector $v \in \mathbb{C}^{n}$, its negative support is the set of indices

$$
\operatorname{nsupp}(v)=\left\{i \in\{1, \ldots, n\} \mid v_{i} \in \mathbb{Z}_{<0}\right\}
$$

A vector $v \in \mathbb{C}^{n}$ has minimal negative support if $\operatorname{nsupp}(v)$ does not properly contain $\operatorname{nsupp}(v+u)$ for any nonzero $u \in \operatorname{ker}_{\mathbb{Z}}(A)$. We denote

$$
N_{v}=\left\{u \in \operatorname{ker}_{\mathbb{Z}}(A) \mid \operatorname{nsupp}(u+v)=\operatorname{nsupp}(v)\right\} .
$$

When $\beta$ is arbitrary, the fake exponents of $H_{A}(\beta)$ with respect to a weight vector $w$ can have negative integer coordinates. For such a $v$, we wish to construct an associated basic Nilsson solution of $H_{A}(\beta)$, in the same way as we did in Proposition 4.3.

Proposition 5.2. Let $w$ be a weight vector for $H_{A}(\beta)$ and let $v \in \mathbb{C}^{n}$ be a fake exponent of $H_{A}(\beta)$ with respect to $w$. The series

$$
\phi_{v}=\sum_{u \in N_{v}} \frac{[v]_{u_{-}}}{[u+v]_{u_{+}}} x^{u+v},
$$

where $[v]_{u_{-}}$and $[u+v]_{u_{+}}$are as in (4.1), is well defined. This series is a formal solution of $H_{A}(\beta)$ if and only if $v$ has minimal negative support, and in that case, $\phi_{v}$ is a basic Nilsson solution of $H_{A}(\beta)$ in the direction of $w$. Consequently, $v$ is an exponent of $H_{A}(\beta)$ with respect to $w$.

Proof. The series is well defined because, as the summation is over $N_{v}$, there cannot be any zeros in the denominators of the summands. The second assertion holds with the same proof as Proposition 3.4.13 of [24]. To see that $\phi_{v}$ is a basic Nilsson solution of $H_{A}(\beta)$, we can argue in the same way as in the proof of Proposition 4.3.

Lemma 3.4.12 of [24] shows that if the negative support of $v$ is empty, then the series (5.2) and (4.3) coincide.

We now consider Nilsson series in the direction of a weight vector.

Definition 5.3. Formal solutions of $H_{A}(\beta)$ of the form $(2.1)$ that satisfy the first two conditions in Definition 2.6 are called (formal) series solutions of $H_{A}(\beta)$ in the direction of $w$.

The $\mathbb{C}$-vector space of logarithm-free formal $A$-hypergeometric series with parameter $\beta$ in the direction of $w$ is denoted by $\mathscr{S}_{w}\left(H_{A}(\beta)\right)$. 
Theorem 5.4. Let $w$ be a weight vector for $H_{A}(\beta)$. The set

$$
\left\{\phi_{v} \mid v \text { is an exponent of } H_{A}(\beta) \text { with minimal negative support }\right\}
$$

is a basis for $\mathscr{S}_{w}\left(H_{A}(\beta)\right)$.

The previous result was stated in Display (7) of [23], in the special case when $I_{A}$ is homogeneous. Its proof for the case when $\beta \in \mathbb{Z}^{d}$ appeared in Proposition 4.2 of [8]; we generalize that argument here.

Proof. Linear independence of the proposed basis elements follows from Lemma 2.8, so we need only show that these series $\operatorname{span} \mathscr{S}_{w}\left(H_{A}(\beta)\right)$.

Let $G(x) \in \mathscr{S}_{w}\left(H_{A}(\beta)\right)$, and suppose that $x^{\nu}$ appears in $G$ with nonzero coefficient $\lambda_{\nu} \in \mathbb{C}$. We claim that $\nu$ has minimal negative support. By contradiction, let $u \in \operatorname{ker}_{\mathbb{Z}}(A)$ such that $\operatorname{nsupp}(\nu+u)$ is strictly contained in $\operatorname{nsupp}(\nu)$. This means that there is $1 \leq i \leq n$ such that $\nu_{i} \in \mathbb{Z}_{<0}$ and $\nu_{i}+u_{i} \in \mathbb{N}$. In particular, $u_{i}>0$.

Since $G$ is a solution of $H_{A}(\beta)$, the operator $\partial^{u_{+}}-\partial^{u_{-}} \in I_{A}$ annihilates $G$. Note that $\operatorname{nsupp}(\nu+u) \subset \operatorname{nsupp}(\nu)$ implies that $\partial^{u_{-}} x^{\nu} \neq 0$. Then some term from $\partial^{u_{+}} G$ needs to equal $\lambda_{v} \partial^{u_{-}} x^{\nu}$, which is a nonzero multiple of $x^{\nu-u_{-}}$. But any function $f$ such that $\partial^{u_{+}} f=x^{\nu-u_{-}}$must involve $\log \left(x_{i}\right)$. This produces the desired contradiction.

Fix $\nu$ such that $x^{\nu}$ appears with nonzero coefficient $\lambda_{\nu}$ in $G$, and let $\psi$ be the subseries of $G$ consisting of terms of the form $\lambda_{\nu+u} x^{\nu+u}$ with $u \in \operatorname{ker}_{\mathbb{Z}}(A)$ and $\lambda_{\nu+u} \in \mathbb{C}$, $\operatorname{such}$ that $\operatorname{nsupp}(\nu+u)=\operatorname{nsupp}(\nu)$. Our goal is to show that $\psi$ is a constant multiple of one of the series from (5.3). This will conclude the proof.

We claim that $\psi$ is a solution of $H_{A}(\beta)$. That the Euler operators $\langle E-\beta\rangle$ annihilate $\psi$ follows since they annihilate every term of $G$. To deal with the toric operators, recall that $\partial^{u_{+}} G=\partial^{u_{-}} G$ for all $u \in \operatorname{ker}_{\mathbb{Z}}(A)$. But terms in $\partial^{u_{+}} G$ that come from $\psi$ can only be matched by terms in $\partial^{u} G$ that also come from $\psi$, so $\partial^{u_{+}}-\partial^{u_{-}}$must annihilate $\psi$, for all $u \in \operatorname{ker}_{\mathbb{Z}}(A)$.

Since $G$ is a solution of $H_{A}(\beta)$ in the direction of $w$, so is $\psi$. Therefore $\operatorname{in}_{w}(\psi)$ is a logarithm-free solution of $\operatorname{in}_{(-w, w)}\left(H_{A}(\beta)\right)$. Theorems 2.3.9 and 2.3.11 in [24] imply that $\operatorname{in}_{w}(\psi)$ is a linear combination of (finitely many) monomial functions arising from exponents of $H_{A}(\beta)$ with respect to $w$. By construction of $\psi$, these exponents differ by elements of $\operatorname{ker}_{\mathbb{Z}}(A)$. Arguing as in the proof of Theorem 3.4.14 in [24], we see that $\operatorname{in}_{w}(\psi)$ can only have one term, that is, $\operatorname{in}_{w}(\psi)=\lambda_{v} x^{v}$, where $v$ is an exponent of $H_{A}(\beta)$ with respect to $w$ and $\lambda_{v} \neq 0$. Since $\lambda_{v} x^{v}$ is a term in $G, v$ has minimal negative support. Thus, $v$ is an exponent of $H_{A}(\beta)$ with respect to $w$ that has minimal negative support.

To finish the proof, we show that $\psi=\lambda_{v} \phi_{v}$. Suppose that $u \in N_{v}$, which means that $u \in \operatorname{ker}_{\mathbb{Z}}(A)$ and $\operatorname{nsupp}(v+u)=\operatorname{nsupp}(v)$. The equality of negative supports implies that $\partial^{u_{-}} x^{v}=[v]_{u_{-}} x^{v-u_{-}}$is nonzero. Since $\partial^{u_{-}} \psi=\partial^{u_{+}} \psi, \partial^{u_{+}} \psi$ must contain the term $\lambda_{v}[v]_{u_{-}} x^{v-u_{-}}$, which can only come from $\partial^{u+} \lambda_{v+u} x^{v+u}$. Thus

$$
\lambda_{v}[v]_{u_{-}} x^{v-u_{-}}=\partial^{u_{+}} \lambda_{v+u} x^{v+u}=\lambda_{v+u}[v+u]_{u_{+}} x^{v+u-u_{+}} .
$$

Consequently $\lambda_{v+u}=\lambda_{v} \frac{[v]_{u_{-}}}{[v+u]_{u_{+}}}$, that is, the coefficient of $x^{v+u}$ in $\psi$ equals $\lambda_{v}$ times the coefficient of $x^{v+u}$ in $\phi_{v}$. Therefore $\psi=\lambda_{v} \phi_{v}$, as we wanted. 
The next theorem gives a bijective map between the space of logarithm-free series solutions of $H_{A}(\beta)$ in the direction of $w$ and a subspace of the logarithmfree solutions of $H_{\rho(A)}\left(\beta_{0}, \beta\right)$ in the direction of $(0, w)$. Note that $\mathscr{S}_{w}\left(H_{A}(\beta)\right) \subseteq$ $\mathcal{N}_{w}\left(H_{A}(\beta)\right)$ follows immediately from the previous result, as the series $\phi_{v}$ are basic Nilsson solutions of $H_{A}(\beta)$ in the direction of $w$.

Theorem 5.5. Let $w$ be a weight vector for $H_{A}(\beta)$ and let $\beta_{0}$ be a homogenizing value for $A, \beta$, and $w$. Then $\rho\left(\mathscr{S}_{w}\left(H_{A}(\beta)\right)\right)$ equals the $\mathbb{C}$-linear span of the logarithm-free basic Nilsson solutions of $H_{\rho(A)}\left(\beta_{0}, \beta\right)$ in the direction of $(0, w)$ which are associated to standard pairs of $\operatorname{in}_{(0, w)}\left(I_{\rho(A)}\right)$ that pass through zero.

Proof. Since $\rho$ is linear, we only need to consider the image of the elements of a basis for the space $\mathscr{S}_{w}\left(H_{A}(\beta)\right)$, such as the one given in Theorem 5.4.

We claim that if $v$ is a fake exponent of $H_{A}(\beta)$ with respect to $w$ that has minimal negative support, then $\rho_{\beta_{0}}(v)=\left(\beta_{0}-|v|, v\right)$ is a fake exponent of $H_{\rho(A)}\left(\beta_{0}, \beta\right)$ with respect to $(0, w)$ corresponding to a standard pair that passes though zero, and moreover, $\rho_{\beta_{0}}(v)$ has minimal negative support. The first part is proved using Lemma 4.4. To see that $\rho_{\beta_{0}}(v)$ has minimal negative support, first recall that $\beta_{0}-|v| \notin \mathbb{Z}$ because $\beta_{0}$ is a homogenizing value for $A, \beta$ and $w$. This implies that 0 is not in the negative support of $\rho_{\beta_{0}}(v)+\mu$ for any $\mu \in \operatorname{ker}_{\mathbb{Z}}(\rho(A))$. Now use the bijection $u \mapsto(-|u|, u)$ between $\operatorname{ker}_{\mathbb{Z}}(A)$ and $\operatorname{ker}_{\mathbb{Z}}(\rho(A))$ and the fact that $v$ has minimal negative support to conclude that $\rho_{\beta_{0}}(v)$ has minimal negative support.

To complete the proof, we show that $\rho\left(\phi_{v}\right)=\phi_{\rho_{\beta_{0}}(v)}$. Lemma 4.7 is this statement in the case when $\operatorname{nupp}(v)=\emptyset$, but now we have to pay attention to the supports of these series.

The same argument we used to check that $\rho_{\beta_{0}}(v)$ has minimal negative support yields $N_{v}=\pi\left(N_{\rho_{\beta_{0}}(v)}\right)$, where $\pi$ is the projection onto the last $n$ coordinates, and therefore $\rho\left(\phi_{v}\right)$ and $\phi_{\rho_{\beta_{0}}(v)}$ have the same support. The verification that the corresponding coefficients are the same is straightforward.

\section{Convergence of hypergeometric Nilsson series}

Until now, we have made no convergence considerations in our study of Nilsson solutions of $A$-hypergeometric systems. The purpose of this section is to investigate convergence issues in detail. In particular, Theorem 6.4 states that, if $w$ is a perturbation of $(1, \ldots, 1)$, the elements of $\mathcal{N}_{w}\left(H_{A}(\beta)\right)$ have a common domain of convergence. Moreover, assuming that the cone spanned by the columns of $A$ is strongly convex, results from Section 7 imply that $\operatorname{dim}_{\mathbb{C}}\left(\mathcal{N}_{w}\left(H_{A}(\beta)\right)\right)=\operatorname{rank}\left(H_{A}(\beta)\right)$. This provides an explicit construction for the space of (multivalued) holomorphic solutions of $H_{A}(\beta)$ in a particular open subset of $\mathbb{C}^{n}$.

When the parameter $\beta$ is generic and $w$ is a perturbation of $(1, \ldots, 1)$, the convergence of the elements of $\mathcal{N}_{w}\left(H_{A}(\beta)\right)$ was shown in [22]. In Subsection 6.2 we complete this study by considering other weight vectors.

Notation 6.1. We have already used the notation $|\cdot|$ to mean the coordinate sum of a vector. When applied to a monomial, such as $x^{u},|\cdot|$ means complex 
absolute value. Let $w$ be a weight vector for $H_{A}(\beta)$ and let $\left\{\gamma_{1}, \ldots, \gamma_{n-d}\right\} \subset \mathbb{Z}^{n}$ be a $\mathbb{Z}$-basis for $\operatorname{ker}_{\mathbb{Z}}(A)$ such that $\gamma_{i} \cdot w>0$ for $i=1, \ldots, n-d$. For any $\varepsilon=\left(\varepsilon_{1}, \ldots, \varepsilon_{n-d}\right) \in \mathbb{R}_{>0}^{n-d}$, we define the (non-empty) open set

$$
\mathscr{U}_{w, \varepsilon}=\left\{x \in \mathbb{C}^{n}|| x^{\gamma_{i}} \mid<\varepsilon_{i} \text { for } i=1, \ldots, n-d\right\} .
$$

\subsection{General parameters}

The following result is the main technical tool in this section.

Theorem 6.2. Let $w$ be a weight vector for $H_{A}(\beta)$ and let $\left\{\gamma_{1}, \ldots, \gamma_{n-d}\right\}$ be a basis for $\operatorname{ker}_{\mathbb{Z}}(A)$ such that $w \cdot \gamma_{i}>0$ for $i=1, \ldots, n-d$. Let $\phi=\sum x^{v+u} p_{u}(\log (x))$ be a basic Nilsson solution of $H_{A}(\beta)$ in the direction of $w$ as in (2.1), such that $|u| \geq 0$ for almost all $u \in \operatorname{supp}(\phi)$, meaning that the set $\{u \in \operatorname{supp}(\phi)|| u \mid<0\}$ is finite. Then there exists $\varepsilon \in \mathbb{R}_{>0}^{n-d}$ such that $\phi$ converges in the open set $\mathscr{U}_{w, \varepsilon}$.

Proof. We may assume without loss of generality that $|u| \geq 0$ for all $u \in \operatorname{supp}(\phi)$. Choose $\beta_{0}$ a homogenizing value for $A, \beta$ and $w$, and recall from Definition 3.16 that the homogenization of $\phi$ is

$$
\rho(\phi)=\sum_{u \in \operatorname{supp}(\phi)} \partial_{0}^{|u|}\left[x_{0}^{\beta_{0}-|v|} x^{v+u} \widehat{p_{u}}\left(\log \left(x_{0}\right), \log \left(x_{1}\right), \ldots, \log \left(x_{n}\right)\right)\right],
$$

where $p_{u}$ and $\widehat{p_{u}}$ are related by (3.2). By Theorem 3.17, $\rho(\phi)$ is a basic Nilsson solution of $H_{\rho(A)}\left(\beta_{0}, \beta\right)$ in the direction of $(0, w)$. Since $H_{\rho(A)}\left(\beta_{0}, \beta\right)$ has regular singularities, Theorem 2.5.16 in [24] implies that there exists $\varepsilon \in \mathbb{R}_{>0}^{n-d}$ such that $\rho(\phi)$ converges (absolutely) in the open set

$$
\mathscr{U}_{(0, w), \varepsilon}=\left\{\left(x_{0}, x\right) \in \mathbb{C}^{n+1}|| x_{0}^{-\left|\gamma_{i}\right|} x^{\gamma_{i}} \mid<\varepsilon_{i}, i=1, \ldots, n-d\right\} .
$$

We make use of the convergence of $\rho(\phi)$ to prove convergence for $\phi$.

As $\rho(\phi)$ converges absolutely in $\mathscr{U}_{(0, w), \varepsilon}$, convergence is preserved when we reorder terms. Use the fact that $|u| \geq 0$ for all $u \in \operatorname{supp}(\phi)$ to rewrite

$$
\rho(\phi)=\sum_{m=0}^{\infty} \partial_{0}^{m}\left[x_{0}^{\beta_{0}-|v|} f_{m}\right]
$$

where

$$
f_{m}\left(x_{0}, \ldots, x_{n}\right)=\sum_{|u|=m} x^{v+u} \widehat{p_{u}}\left(\log \left(x_{0}\right), \log \left(x_{1}\right), \ldots, \log \left(x_{n}\right)\right)
$$

is a polynomial in $\log \left(x_{0}\right)$ whose coefficients are (multivalued) holomorphic functions of the $n$ variables $x_{1}, \ldots, x_{n}$. Recall that, by Definition 2.6, there exists a positive integer $K$ such that the degree of $f_{m}$ in $\log \left(x_{0}\right)$ is less than or equal to $K$ for all $m \in \mathbb{N}$. A key observation is that

$$
\left.\sum_{m=0}^{\infty}\left(x_{0}^{\beta_{0}-|v|-m} f_{m}\left(x_{0}, x_{1}, \ldots, x_{n}\right)\right)\right|_{x_{0}=1}=\phi\left(x_{1}, \ldots, x_{n}\right) .
$$

Since $\{1\} \times \mathscr{U}_{w, \varepsilon} \subset \mathscr{U}_{(0, w), \varepsilon}$, if we show that $\sum_{m=0}^{\infty} x_{0}^{\beta_{0}-|v|-m} f_{m}$ converges absolutely on $\mathscr{U}_{(0, w), \varepsilon}$, the convergence of $\phi$ on $\mathscr{U}_{w, \varepsilon}$ will follow. 
For $\lambda \in \mathbb{C}$ and $m \in \mathbb{N}$, we denote the $m$-th descending factorial by

$$
[\lambda]_{m}=\lambda(\lambda-1) \ldots(\lambda-m+1) .
$$

Set $\lambda=\beta_{0}-|v|$. Since $\beta_{0}$ is a homogenizing value for $A, \beta$ and $w$, we have $\lambda \notin \mathbb{Z}$. We claim that the domain of convergence of $\sum_{m=0}^{\infty}[\lambda]_{m} x_{0}^{\lambda-m} f_{m}$ contains $\mathscr{U}_{(0, w), \varepsilon}$. But if this is true, the convergence of $\sum_{m=0}^{\infty} x_{0}^{\lambda-m} f_{m}$ on $\mathscr{U}_{(0, w), \varepsilon}$ follows by comparison, since the absolute value of $[\lambda]_{m}$ grows like $(m-1)$ ! as $m$ goes to $\infty$. Thus, all we need to show in order to finish our proof is that $\sum_{m=0}^{\infty}[\lambda]_{m} x_{0}^{\lambda-m} f_{m}$ converges absolutely on $\mathscr{U}_{(0, w), \varepsilon}$.

Consider $f_{m}$ as a polynomial in $\log \left(x_{0}\right)$. By construction, the coefficients of $f_{m}$ are constant with respect to $x_{0}$. Denote by $f_{m}^{(r)}$ the $r$-th derivative of $f_{m}$ with respect to $\log \left(x_{0}\right)$. Then $f_{m}^{(K+1)}=0$ since the degree of $f_{m}$ in $\log \left(x_{0}\right)$ is at most $K$. We compute $\partial_{0}^{m}\left(x_{0}^{\lambda} f_{m}\right)$ for $m \geq K$, using the fact that $f_{m}^{(r)}=0$ if $r>K$.

$$
\begin{aligned}
\partial_{0}^{m}\left(x_{0}^{\lambda} f_{m}\right) & =\partial_{0}^{m-1} \partial_{0}\left(x_{0}^{\lambda} f_{m}\right)=\partial_{0}^{m-1}\left(x_{0}^{\lambda-1}\left(\alpha f_{m}+f_{m}^{\prime}\right)\right) \\
& =\partial_{0}^{m-2}\left(x_{0}^{\lambda-2}\left(\lambda(\lambda-1) f_{m}+(\lambda+(\lambda-1)) f_{m}^{\prime}+f_{m}^{\prime \prime}\right)\right) \\
& =\cdots \\
& =x_{0}^{\lambda-m}\left(c_{0}(\lambda, m) f_{m}+c_{1}(\lambda, m) f_{m}^{\prime}+\ldots+c_{K}(\lambda, m) f_{m}^{(K)}\right),
\end{aligned}
$$

where

$$
c_{j}(\lambda, m)=\sum_{1 \leq i_{1}<\cdots<i_{m-j} \leq m} \prod_{k=1}^{j}\left(\lambda-i_{k}+1\right) .
$$

Note that, as $m$ goes to $\infty$, the dominant term in absolute value in $c_{j}(\lambda, m)$ is $\prod_{r=j}^{m-1}(\lambda-r+1)$, which grows like $\prod_{r=j}^{m-1} r=\frac{(m-1) !}{(j-1) !}$. But then, if $j>0,[\lambda-j]_{m}$ grows faster than $c_{j}(\alpha, m)$ as $m$ goes to $\infty$, because $[\lambda-j]_{m}$ grows like $\frac{(m+j-1) !}{(j-1) !}$. In other words,

$$
\lim _{m \rightarrow \infty} \frac{c_{j}(\lambda, m)}{[\lambda-j]_{m}}=0 \quad \text { for } j \geq 1 .
$$

Since $\rho(\phi)$ converges absolutely on the open set $\mathscr{U}_{(0, w), \varepsilon}, \partial_{0} \rho(\phi)$ is also absolutely convergent on $\mathscr{U}_{(0, w), \varepsilon}$, and

$$
\begin{aligned}
\partial_{0} \rho(\phi) & =\partial_{0} \sum_{m=0}^{\infty} \partial_{0}^{m}\left[x_{0}^{\lambda} f_{m}\right]=\sum_{m=0}^{\infty} \partial_{0}^{m}\left[\partial_{0} x_{0}^{\lambda} f_{m}\right]=\sum_{m=0}^{\infty} \partial_{0}^{m}\left[\lambda x_{0}^{\lambda-1} f_{m}+x_{0}^{\lambda} f_{m}^{\prime}\right] x_{0}^{-1} \\
& =\lambda \sum_{m=0}^{\infty} \partial_{0}^{m}\left[x_{0}^{\lambda-1} f_{m}\right]+\sum_{m=0}^{\infty} \partial_{0}^{m}\left[x_{0}^{\lambda-1} f_{m}^{\prime}\right] .
\end{aligned}
$$

The series $\sum_{m=0}^{\infty} \partial_{0}^{m}\left[x_{0}^{\lambda-1} f_{m}\right]$ converges in $\mathscr{U}_{(0, w), \varepsilon}$ because it is a basic Nilsson solution of the regular hypergeometric system $H_{\rho(A)}\left(\beta_{0}-1, \beta\right)$ in the direction of $(0, w)$. (We may need to decrease $\varepsilon$ coordinatewise for the previous assertion to 
hold.) This, and the convergence of $\partial_{0}(\rho(\phi))$, imply that $\sum_{m=0}^{\infty} \partial_{0}^{m}\left[x_{0}^{\lambda-1} f_{m}^{\prime}\right]$ converges absolutely on $\mathscr{U}_{(0, w), \varepsilon}$. Proceeding by induction, we conclude that

$$
\sum_{m=0}^{\infty} \partial_{0}^{m} x_{0}^{\lambda-j} f_{m}^{(j)} \quad \text { converges absolutely on } \mathscr{U}_{(0, w), \varepsilon} \text { for } j=1, \ldots, K .
$$

Now we induct on $K-\ell$ to show that

$$
\sum_{m=0}^{\infty} x_{0}^{\lambda-m-\ell}[\lambda-\ell]_{m} f_{m}^{(\ell)} \text { converges absolutely on } \mathscr{U}_{(0, w), \varepsilon} \text { for } \ell=0,1, \ldots, K .
$$

The $\ell=0$ case of this assertion is exactly what we needed to verify in order to finish the proof.

If $K-\ell=0$, then $f_{m}^{(\ell)}=f_{m}^{(K)}$ is a (maybe zero) constant with respect to $x_{0}$, and therefore (6.5) is the $j=K$ case of (6.4). For the inductive step, we compute the $m$-th derivative inside the series. The sum $\sum_{m=0}^{\infty} \partial_{0}^{m} x_{0}^{\lambda-\ell} f_{m}^{(\ell)}$ equals

$$
\begin{aligned}
& \sum_{m=0}^{\infty} x_{0}^{\lambda-\ell-m}\left([\lambda-\ell]_{m} f_{m}^{(\ell)}+c_{1}(\lambda-\ell, m) f_{m}^{(\ell+1)}+\cdots+c_{K-\ell}(\lambda-\ell, m) f_{m}^{(K)}\right) \\
& \quad=\sum_{m=0}^{\infty} x_{0}^{\lambda-\ell-m}[\lambda-\ell]_{m} f_{m}^{(\ell)} \\
& \quad+\sum_{m=0}^{\infty} x_{0}^{\lambda-\ell-m} c_{1}(\lambda-\ell, m) f_{m}^{(\ell+1)}+\cdots+\sum_{m=0}^{\infty} x_{0}^{\lambda-\ell-m} c_{K-\ell}(\lambda-\ell, m) f_{m}^{(K)} .
\end{aligned}
$$

We want to show that $\sum_{m=0}^{\infty} x_{0}^{\lambda-\ell-m}[\lambda-\ell]_{m} f_{m}^{(\ell)}$ converges absolutely on $\mathscr{U}_{(0, w), \varepsilon}$. We know that $\sum_{m=0}^{\infty} \partial_{0}^{m} x_{0}^{\lambda-\ell} f_{m}^{(\ell)}$ converges absolutely on $\mathscr{U}_{(0, w), \varepsilon}$ by $(6.4)$, so we need to control the other summands. But the inductive hypothesis tells us that (6.5) is true for $\ell+1, \ldots, K$. By comparison using (6.3), and harmlessly multiplying by $x_{0}^{j}$, we conclude that the series $\sum_{m=0}^{\infty} x_{0}^{\lambda-\ell-m} c_{j}(\lambda-\ell, m) f_{m}^{(\ell+j)}$ converges absolutely on $\mathscr{U}_{(0, w), \varepsilon}$ for $1 \leq j<K-\ell$.

Corollary 6.3. If $w$ is a weight vector for $H_{A}(\beta)$ which is a perturbation of $(1, \ldots, 1)$, then all the basic Nilsson solutions of $H_{A}(\beta)$ in the direction of $w$ have a common (open) domain of convergence.

Proof. Let $w$ be a weight vector which is a perturbation of $(1, \ldots, 1)$. Recall that, if $\phi=x^{v} \sum_{u \in C} x^{u} p_{u}(\log (x))$ is a basic Nilsson solution of $H_{A}(\beta)$ in the direction of $w$, then $|u| \geq 0$ for $u \in C$ (see the paragraph after Definition 3.3). Now apply Theorem 6.2.

One of the main objectives of this article was to construct a basis of series solutions of $H_{A}(\beta)$ that have a common domain of convergence. While such constructions are well known in the regular case, when $I_{A}$ is inhomogeneous, important theoretical tools become unavailable. A way of bypassing this difficulty is to assume that the parameters are generic and $w$ is a perturbation of $(1, \ldots, 1)$ as in [22]. 
The following result gives the desired construction, without any assumptions on $\beta$ (but with the same assumption on $w$ ). Its proof can be found in Section 7 , after Theorem 7.5.

Theorem 6.4. Assume that the cone over the columns of $A$ is strongly convex, and let $w$ be a weight vector for $H_{A}(\beta)$ that is a perturbation of $(1, \ldots, 1)$. Then

$$
\operatorname{dim}_{\mathbb{C}}\left(\mathcal{N}_{w}\left(H_{A}(\beta)\right)\right)=\operatorname{rank}\left(H_{A}(\beta)\right)
$$

and there exists $\varepsilon \in \mathbb{R}_{>0}^{n-d}$ such that every element of $\mathcal{N}_{w}\left(H_{A}(\beta)\right)$ converges in the open set $\mathscr{U}_{w, \varepsilon}$.

\subsection{Generic parameters}

In this subsection, we assume that $\beta$ is generic as in Convention 4.2. In this case, by Theorem 4.8 , the set

$$
\mathscr{B}_{w}=\left\{\phi_{v} \mid v \text { is an exponent of } H_{A}(\beta) \text { with respect to } w\right\}
$$

is a basis for $\mathcal{N}_{w}\left(H_{A}(\beta)\right)$, and we can write the series $\phi_{v}$ as in (4.3), by the genericity of the parameters.

We wish to determine which elements of $\mathscr{B}_{w}$ have an open domain of convergence. This depends on the choice of the weight vector $w$.

Theorem 6.5. Let $\beta$ be generic, let $w$ be a weight vector for $H_{A}(\beta)$, and let $\phi_{v} \in \mathscr{B}_{w}$. There exists $\varepsilon \in \mathbb{R}_{>0}^{n-d}$ such that $\phi_{v}$ converges in $\mathscr{U}_{w, \varepsilon}$ if and only if $|u| \geq 0$ for almost all $u \in \operatorname{supp}\left(\phi_{v}\right)$.

Proof. If $|u| \geq 0$ for almost all $u \in \operatorname{supp}\left(\phi_{v}\right)$, then $\phi_{v}$ converges on an open set $\mathscr{U}_{w, \varepsilon}$ by Theorem 6.2 .

Now assume that there exist an infinite number of elements $u \in \operatorname{supp}\left(\phi_{v}\right)$ such that $|u|<0$. Using the description of $\operatorname{supp}\left(\phi_{v}\right)$ from (4.3), which applies when $\beta$ is generic, we can find $\nu \in \operatorname{supp}\left(\phi_{v}\right)$ such that $|\nu|<0$ and $\left\{m \nu \mid m \geq m_{0} \in \mathbb{N}\right\} \subset$ $\operatorname{supp}\left(\phi_{v}\right)$ for some $m_{0} \in \mathbb{N}$.

Let $\psi$ be the subseries of $\phi_{v}$ whose terms are indexed by the set $\{m \nu \mid m \geq$ $\left.m_{0} \in \mathbb{N}\right\}$. The coefficient of $x^{v+m \nu}$ in $\psi$ is

$$
\frac{\prod_{\nu_{i}<0} \prod_{j=1}^{-m \nu_{i}}\left(v_{i}-j+1\right)}{\prod_{\nu_{i} \geq 0} \prod_{j=1}^{m \nu_{i}}\left(v_{i}+j\right)}
$$

which grows like $\lambda_{m}=\prod_{\nu_{i}<0}\left(-\nu_{i} m\right) ! / \prod_{\nu_{i}>0}\left(\nu_{i} m\right)$ ! as $m$ goes to $\infty$. Since $|\nu|<0$, $\lim _{m \rightarrow \infty} \lambda_{m}=\infty$. Therefore $\psi$ cannot converge absolutely unless $x_{i}=0$ for some $i$ such that $\nu_{i}>0$, and consequently $\phi_{v}$ does not have an open domain of convergence.

Remark 6.6. In this section we study convergence of Nilsson solutions of $H_{A}(\beta)$ in the direction of a weight vector $w$. We can change the point of view and fix a basis $\left\{\gamma_{1}, \ldots, \gamma_{n-d}\right\}$ of $\operatorname{ker}_{\mathbb{Z}}(A)$; then our results apply to any weight vector $w$ such that $\gamma_{i} \cdot w>0$ for $i=1, \ldots, n-d$. 
Since $\beta$ is generic, all the information necessary to compute the Nilsson solutions of $H_{A}(\beta)$ associated to a weight vector $w$ can be extracted from the top-dimensional standard pairs of $\operatorname{in}_{(0, w)}\left(I_{\rho(A)}\right)$; the simplices appearing in these standard pairs are the maximal simplices of the coherent triangulation $\Delta_{(0, w)}$ of $\rho(A)$. These triangulations also control the possible regions of convergence of basic Nilsson solutions of $H_{A}(\beta)$ in the direction of $w$, as a change in triangulation changes $\mathscr{U}_{w, \varepsilon}$. There is an object that parametrizes all coherent triangulations of the configuration $\rho(A)$. This object is called secondary fan of $\rho(A)$, and was introduced by Gel'fand, Kapranov and Zelevinsky (see Chapter 7 of [12]).

To construct the secondary fan of $\rho(A)$, we need a Gale dual for $\rho(A)$, that is, a matrix $B \in \mathbb{Z}^{(n-d) \times(n+1)}$ whose columns form a basis for $\operatorname{ker}_{\mathbb{Z}}(\rho(A))$. Denote by $b_{0}, \ldots, b_{n}$ the rows of $B$, and let $\Delta$ be a coherent triangulation of $\rho(A)$. For each maximal simplex $\sigma \in \Delta$, we define a cone

$$
\mathscr{K}_{\sigma}=\left\{\sum_{i \notin \sigma} \lambda_{i} b_{i} \mid \lambda_{i} \geq 0\right\} .
$$

Note that the set $\left\{b_{i} \mid i \notin \sigma\right\}$ is linearly independent by Lemma 7.1.16 in [12], and therefore $\mathscr{K}_{\sigma}$ is full-dimensional. Define $\mathscr{K}_{\Delta}=\cap_{\sigma \in \Delta} \mathscr{K}_{\sigma}$. Then $\left(w_{0}, w\right) \in \mathbb{R}^{n+1}$ is such that $\Delta_{\left(w_{0}, w\right)}=\Delta$ if and only if $\left(w_{0}, w\right) \cdot B$ belongs to the interior $\mathscr{K}_{\Delta}^{\circ}$ of $\mathscr{K}_{\Delta}$. The cones $\mathscr{K}_{\Delta}$ for all coherent triangulations $\Delta$ of $\rho(A)$ are the maximal cones in a polyhedral fan, called the secondary fan of $\rho(A)$ (see also Theorem 7.1.17 in [12]).

Given generic $\beta$, fix a weight vector $w$ for $H_{A}(\beta)$. Then the supports of the basic Nilsson solutions of $H_{A}(\beta)$ in the direction of $w$ can be described by means of the cones associated to maximal simplices of the triangulation $\Delta_{(0, w)}$ of $\rho(A)$. Indeed, if $\left(v_{0}, v\right)$ is the exponent associated to a standard pair $\left(\partial^{\alpha}, \sigma\right)$ of the monomial ideal $\operatorname{in}_{(0, w)}\left(I_{\rho(A)}\right)$, so that $\sigma \in \Delta_{(0, w)}$ and $0 \in \sigma$, we know that the support of the dehomogenized series $\phi_{v}$ is

$$
\operatorname{supp}\left(\phi_{v}\right)=\left\{u \in \operatorname{ker}_{\mathbb{Z}}(A) \mid u_{i}+v_{i} \geq 0 \quad \forall i \notin \sigma\right\} .
$$

Note that, as $0 \in \sigma$, the zeroth row of the Gale dual $B$ is not present in this description. Since the columns of $B$ span $\operatorname{ker}_{\mathbb{Z}}(\rho(A))$, the support of $\phi_{v}$ is naturally identified with

$$
\operatorname{supp}\left(\phi_{v}\right)=\left\{\left(b_{1} \cdot \nu, \ldots, b_{n} \cdot \nu\right) \mid \nu \in \mathbb{Z}^{n-d} \text { and } \nu \cdot b_{i} \geq-v_{i}, i \notin \sigma\right\} .
$$

Theorem 6.7 gives a combinatorial condition for a series $\phi_{v}$ associated to a cone $\mathscr{K}_{\sigma}$ (that is, to a standard pair $\left.\left(\partial^{\alpha}, \sigma\right)\right)$ to have an open domain of convergence. Note that several series may be associated with a single cone, and some of them may have open domains of convergence, while others do not, see Example 6.8.

Given $w$ a weight vector for $H_{A}(\beta)$, let $\left\{\left(-\left|\gamma_{1}\right|, \gamma_{1}\right), \ldots,\left(-\left|\gamma_{n-d}\right|, \gamma_{n-d}\right)\right\}$ be a $\mathbb{Z}$-basis of $\operatorname{ker}_{\mathbb{Z}}(\rho(A))$ such that for any $i=1, \ldots, n-d$, we have $\gamma_{i} \cdot w>0$. The vectors in this basis are the columns of a Gale dual matrix of $\rho(A)$, whose rows we denote by $b_{0}, \ldots, b_{n}$.

Theorem 6.7. Let $\beta$ be generic, let $w$ be a weight vector for $H_{A}(\beta)$, and choose a Gale dual of $\rho(A)$ as above. Let $\left(v_{0}, v\right)$ be an exponent of $\rho(A)$ corresponding to a standard pair $\left(\partial^{\alpha}, \sigma\right)$ of $\operatorname{in}_{(0, w)}\left(I_{\rho(A)}\right)$ that passes through zero. 
- If $-b_{0}$ belongs to the interior of $\mathscr{K}_{\sigma}$, then the series $\phi_{v}$ has an open domain of convergence that contains $\mathscr{U}_{w, \varepsilon}$ for some $\varepsilon \in \mathbb{R}_{>0}^{n-d}$.

- If $-b_{0} \notin \mathscr{K}_{\sigma}$, then the series $\phi_{v}$ does not have an open domain of convergence.

Proof. For the first statement, suppose that $-b_{0} \in \mathscr{K}_{\sigma}^{\circ}$, so that $-b_{0}=\sum_{i \notin \sigma} \lambda_{i} b_{i}$ with $\lambda_{i}>0$. Then the polyhedron $\left\{\nu \in \mathbb{R}^{n-d} \mid \nu \cdot b_{i} \geq-v_{i}, i \notin \sigma,-b_{0} \cdot \nu<0\right\}$ is bounded and can contain only finitely many points with integer coordinates. Using (6.7) and the fact that for $u=\left(b_{1} \cdot \nu, \ldots, b_{n} \cdot \nu\right) \in \operatorname{supp}\left(\phi_{v}\right)$ we have:

$$
|u|=\sum_{i=1}^{n} b_{i} \cdot \nu=-b_{0} \cdot \nu
$$

we see that $|u| \geq 0$ for all but at most finitely many elements of $\operatorname{supp}\left(\phi_{v}\right)$. Now apply Theorem 6.5 to conclude that $\phi_{v}$ has an open domain of convergence of the desired form.

We now prove the second statement. As $\left\{b_{i} \mid i \notin \sigma\right\}$ is a basis of $\mathbb{R}^{n-d}$, we can write $-b_{0}=\sum_{i \notin \sigma} \lambda_{i} b_{i}$. Suppose that $\lambda_{i_{0}}<0$ for some $i_{0} \notin \sigma$, and consider the infinite set

$$
\left\{\nu \in \mathbb{Z}^{n-d} \mid \nu \cdot b_{i}=0 \text { for } i \notin \sigma \cup\left\{i_{0}\right\} \text { and } \nu \cdot b_{i_{0}}>0\right\} \text {. }
$$

For each element $\nu$ of this set, $\left(\nu \cdot b_{1}, \ldots, \nu \cdot b_{n}\right)$ is an element of $\operatorname{supp}\left(\phi_{v}\right)$, and the sum of its coordinates is

$$
\sum_{i=1}^{n} \nu \cdot b_{i}=\nu \cdot\left(\sum_{i=1}^{n} b_{i}\right)=\nu \cdot\left(-b_{0}\right)=\nu \cdot\left(\sum_{i \notin \sigma} \lambda_{i} b_{i}\right)=\lambda_{i_{0}}\left(\nu \cdot b_{i_{0}}\right) .
$$

Thus, $\operatorname{supp}\left(\phi_{v}\right)$ has an infinite subset consisting of vectors whose coordinate sum is negative. By Theorem 6.5, $\phi_{v}$ does not have an open domain of convergence.

Example 6.8. If $-b_{0}$ is in the boundary of $\mathscr{K}_{\sigma}$, a series arising from $\sigma$ may or may not have an open domain of convergence, depending on the specific standard pair to which it belongs. For instance, consider:

$$
A=\left[\begin{array}{rrrr}
0 & 1 & 0 & -1 \\
1 & 0 & 2 & 4
\end{array}\right]
$$

Then the convex hull of $\rho(A)$ is a triangle of normalized volume 4 . For the weight $(0, w)=(0,1,1,3,3)$, the corresponding triangulation of $\rho(A)$ has only one maximal simplex, $\operatorname{conv}(\rho(A))$ itself. In this case,

$$
I_{\rho(A)}=\left\langle\partial_{3}^{2}-\partial_{2} \partial_{4}, \partial_{1}^{2}-\partial_{0} \partial_{3}\right\rangle \quad \text { and } \quad \operatorname{in}_{(0, w)}\left(I_{\rho(A)}\right)=\left\langle\partial_{0} \partial_{3}, \partial_{1}^{4}, \partial_{1}^{2} \partial_{3}, \partial_{3}^{2}\right\rangle .
$$

The monomial ideal $\operatorname{in}_{(0, w)}\left(I_{\rho(A)}\right)$ has four top-dimensional standard pairs:

$$
(1,\{0,2,4\}), \quad\left(\partial_{1},\{0,2,4\}\right), \quad\left(\partial_{1}^{2},\{0,2,4\}\right) \quad \text { and } \quad\left(\partial_{1}^{3},\{0,2,4\}\right) .
$$


A Gale dual of $\rho(A)$ is

$$
B=\left[\begin{array}{rr}
1 & 0 \\
-2 & 0 \\
0 & -1 \\
1 & 2 \\
0 & -1
\end{array}\right]
$$

and we have $-b_{0}=(1 / 2) b_{1}$, so that $-b_{0}$ is in the boundary of $\mathscr{K}_{\sigma}$ for $\sigma=\{0,2,4\}$, the unique maximal simplex in the triangulation of $\rho(A)$ induced by $(0,1,1,3,3)$.

For a given generic $\beta$ and $0 \leq j \leq 3$, let $\phi_{j}$ denote the logarithm-free series solution of $H_{A}(\beta)$ associated to the standard pair $\left(\partial_{1}^{j}, \sigma\right)$. Then

$$
\operatorname{supp}\left(\phi_{j}\right)=\left\{\begin{array}{l|l}
u=\left(-2 \nu_{1},-\nu_{2}, \nu_{1}+2 \nu_{2},-\nu_{2}\right) & \begin{array}{l}
\nu=\left(\nu_{1}, \nu_{2}\right) \in \mathbb{Z}^{2} \\
-2 \nu_{1} \geq-j, \nu_{1}+2 \nu_{2} \geq 0
\end{array}
\end{array}\right\} .
$$

For $u \in \operatorname{supp}\left(\phi_{j}\right)$ as above, $|u|=-\nu_{1} \geq-j / 2$. Consequently, if $j=0,1,|u| \in \mathbb{Z}$ implies that $|u| \geq 0$ for all $u \in \operatorname{supp}\left(\phi_{j}\right)$, and therefore $\phi_{j}$ has an open domain of convergence by Theorem 6.5 .

If $j=2,3$, consider $\nu=(1, k)$ for $k \in \mathbb{N}$. Then

$$
-2 \nu_{1}=-2 \geq-j \quad \text { and } \quad \nu_{1}+2 \nu_{2}=1+2 k \geq 0 \quad \text { for } k \in \mathbb{N} .
$$

This means that $u=\left(-2 \nu_{1},-\nu_{2}, \nu_{1}+2 \nu_{2},-\nu_{2}\right)=(-2,-k, 1+2 k,-k) \in \operatorname{supp}\left(\phi_{j}\right)$ for $k \in \mathbb{N}$. But then $|u|=-1$ for infinitely many elements of $\operatorname{supp}\left(\phi_{j}\right)$, and therefore $\phi_{j}$ does not have an open domain of convergence.

Geometrically, consider the polyhedron

$$
Q_{j}=\left\{\nu \in \mathbb{R}^{2} \mid-2 \nu_{1} \geq-j, \quad \nu_{1}-2 \nu_{2} \geq 0\right\},
$$

which is a translate of a cone. Let $L$ be the line orthogonal to $-b_{0}=(-1,0)$. Since $L$ contains one of the faces of $Q_{0}$, there are no points in this polyhedron whose dot product with $-b_{0}$ is strictly negative. If $j=1,2,3$, then the set $Q_{j} \cap$ $\left\{\nu \in \mathbb{R}^{2} \mid-b_{0} \cdot \nu<0\right\}$ in unbounded. However, when $j=1$, this set contains no integer points. When $j=2,3, Q_{j} \cap\left\{\nu \in \mathbb{R}^{2} \mid-b_{0} \cdot \nu<0\right\}$ contains infinitely many integer points.

This example also illustrates the fact that, in order to determine whether a basic Nilsson solution of $H_{A}(\beta)$ in the direction of $w$ has an open domain of convergence, knowledge of the triangulation of $\rho(A)$ induced by $(0, w)$ is not sufficient. For instance, note that $(1,1,2,1)$ is a perturbation of $(1,1,1,1)$, so all Nilsson solutions of $H_{A}(\beta)$ in the direction of $(1,1,2,1)$ have an open domain of convergence by Corollary 6.3. However, $(0,1,1,2,1)$ and $(0,1,1,3,3)$ induce the same triangulation of $\rho(A)$, and we already know that there are Nilsson solutions of $H_{A}(\beta)$ in the direction of $(1,1,3,3)$ which do not have open domains of convergence.

As a consequence of Theorem 6.7, we have combinatorial bounds for the dimension of the space of convergent Nilsson solutions of $H_{A}(\beta)$ in the direction of $w$, that hold for generic parameters $\beta$. 
Corollary 6.9. Let $\beta$ be generic, and let $w$ be a weight vector for $H_{A}(\beta)$. Then

$$
\sum_{\sigma \in T} \operatorname{vol}(\sigma) \geq \operatorname{dim}_{\mathbb{C}}\left(\left\{\phi \in \mathcal{N}_{w}\left(H_{A}(\beta)\right) \text { convergent }\right\}\right) \geq \sum_{\sigma \in T_{\circ}} \operatorname{vol}(\sigma),
$$

where

$$
T_{\circ}=\left\{\begin{array}{l|l}
\sigma \text { facet of } \Delta_{(0, w)} \mid \begin{array}{l}
0 \in \sigma \\
-b_{0} \in \mathscr{K}_{\sigma}^{\circ}
\end{array}
\end{array}\right\} ; \quad T=\left\{\begin{array}{ll}
\sigma \text { facet of } \Delta_{(0, w)} \mid \begin{array}{l}
0 \in \sigma \\
-b_{0} \in \mathscr{K}_{\sigma}
\end{array}
\end{array}\right\} \text {. }
$$

The second part of Theorem 6.7 can be restated in a more combinatorial fashion.

Corollary 6.10. Let $\beta$ be generic, and let $w$ be a weight vector for $H_{A}(\beta)$. If an element of the set $\mathscr{B}_{w}$ from (6.6) has an open domain of convergence, then its associated maximal simplex in the triangulation $\Delta_{(0, w)}$ of $\rho(A)$ also belongs to a coherent triangulation of $\rho(A)$ defined by $\left(0, w^{\prime}\right)$, where $w^{\prime}$ is a perturbation of $(1, \ldots, 1)$.

Proof. Using Theorem 6.7 and its notation, we have that, if an element of the set $\mathscr{B}_{w}$ from $(6.6)$ has an open domain of convergence, $-b_{0}=(0,1, \ldots, 1) \cdot B \in \mathscr{K}_{\sigma}$. Therefore, there is a perturbation $w^{\prime}$ of $(1, \ldots, 1)$ with $\sum_{i=1}^{n} w_{i}^{\prime} b_{i} \in \mathscr{K}_{\sigma}^{\circ}$. It follows that $\sigma$ is a maximal simplex in the coherent triangulation of $\rho(A)$ defined by $\left(0, w^{\prime}\right)$.

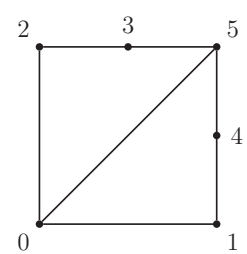

$$
\Delta_{1}=\{\{0,1,5\},\{0,2,5\}\}
$$

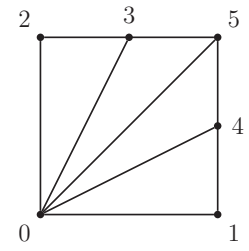

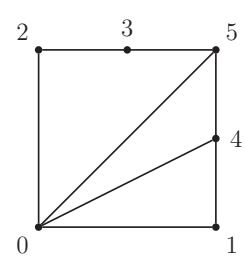

$\Delta_{2}=\{\{0,1,4\},\{0,4,5\},\{0,2,5\}\}$

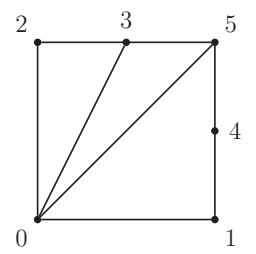

$$
\Delta_{3}=\{\{0,1,4\},\{0,4,5\},\{0,3,5\},\{0,2,3\}\} \quad \Delta_{4}=\{\{0,1,5\},\{0,3,5\},\{0,2,3\}\}
$$

Figure 1. The coherent triangulations of $\rho(A)$ corresponding to perturbations of the vector $(0,1 \ldots, 1)$ for Example 6.11 .

Example 6.11. Corollary 6.10 allows us to decide by inspecting the triangulations of $\rho(A)$, whether $H_{A}(\beta)$ has Nilsson solutions in the direction of a weight vector that do not have an open domain of convergence. Take for instance

$$
A=\left[\begin{array}{lllll}
2 & 0 & 1 & 2 & 2 \\
0 & 2 & 2 & 1 & 2
\end{array}\right]
$$

and consider the coherent triangulations of $\rho(A)$, or, equivalently, the coherent triangulations of $A \cup\{0\}$. Note that the triangulations $\Delta_{1}, \ldots, \Delta_{4}$ appearing in 
Figure 1 are all the triangulations induced by perturbations of $(0,1, \ldots, 1)$ (that is, induced by vectors $\left(0, w^{\prime}\right)$ with $w^{\prime}$ a perturbation of $\left.(1, \ldots, 1)\right)$. Now consider the triangulation $\Delta_{5}$ drawn in Figure 2. The simplex $\{0,3,4\}$ belongs to $\Delta_{5}$ and passes through zero, but does not appear in any triangulation of $\rho(A)$ induced by a perturbation of $(0,1, \ldots, 1)$; therefore, Corollary 6.10 ensures that the corresponding Nilsson solutions of $H_{A}(\beta)$ (for generic $\beta$ ) do not have open domains of convergence.

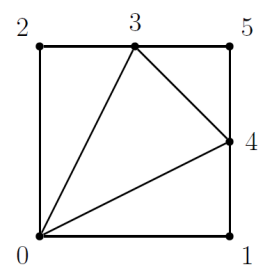

$$
\Delta_{5}=\{\{0,1,4\},\{0,3,4\},\{0,2,3\},\{3,4,5\}\}
$$

Figure 2. A coherent triangulation of $\rho(A)$ from Example 6.11.

\section{The irregularity of $H_{A}(\beta)$ via its Nilsson solutions}

In this section, we give an alternative proof of Corollary 3.16 in [25] using our study of Nilsson solutions of $H_{A}(\beta)$. We assume as in [25] that the columns of $A$ span a strongly convex cone.

In Theorem 3.18 we computed the image of $\rho$. There is one case in which this map is guaranteed to be onto.

Proposition 7.1. Suppose that $w$ is a perturbation of $(1, \ldots, 1)$. Then the map $\rho$ is surjective.

Proof. First note that none of the minimal generators of the monomial ideal $\operatorname{in}_{(0, w)}\left(I_{\rho(A)}\right)$ have $\partial_{0}$ as a factor. If $u \in \mathbb{N}^{n+1}$ and $\partial^{u} \notin \operatorname{in}_{(0, w)}\left(I_{\rho(A)}\right)$, this implies that $\partial_{0}^{k} \partial^{u} \notin \operatorname{in}_{(0, w)}\left(I_{\rho(A)}\right)$ for all $k \in \mathbb{N}$. We conclude that all the standard pairs of $\operatorname{in}_{(0, w)}\left(I_{\rho(A)}\right)$ pass through zero. Theorem 3.18 completes the proof.

We wish to find weight a vector $w$ for $H_{A}(\beta)$ for which $\rho$ is not surjective. We require the following statement.

Lemma 7.2. Let $A \in \mathbb{Z}^{d \times n}$ be of full rank $d$ with columns spannnig a strongly convex cone. If the row span of $A$ does not contain the vector $(1, \ldots, 1)$, there exists $w \in \mathbb{R}_{>0}^{n}$ such that the coherent triangulation $\Delta_{(0, w)}$ of $\rho(A)$ has a maximal simplex that does not pass through zero. Given $\beta \in \mathbb{C}^{d}$, the vector $w$ can be chosen to be a weight vector for $H_{A}(\beta)$.

Proof. We use the description of the secondary fan of $\rho(A)$ from Section 6. Let $B$ be a Gale dual matrix of $\rho(A)$ with rows $b_{0}, \ldots, b_{n}$. Since $(1, \ldots, 1)$ is not in the rowspan of $A$, the zeroth row of $B$ is nonzero. Because $B$ has full rank $n-d$, we can choose $\sigma \subset\{1, \ldots n\}$ of cardinality $d+1$ such that $\left\{b_{i} \mid i \notin \sigma\right\}$ is linearly independent. 
The assumption that the columns $a_{1}, \ldots, a_{n}$ of $A$ span a strongly convex cone means that there exists a vector $h \in \mathbb{R}^{d}$ such that $h \cdot A$ is coordinatewise positive. As $\rho(A) \cdot B=0, \sum_{i=1}^{n}\left(h \cdot a_{i}\right) b_{i}=0$.

Choose $w \in \mathbb{R}_{>0}^{n}$ and positive real $\lambda_{i}$ for $i \notin \sigma$, such that

$$
w_{i}+\lambda_{0}=h \cdot a_{i} \text { for } i \in \sigma, \text { and } w_{i}+\lambda_{0}-\lambda_{i}=h \cdot a_{i} \text { for } i \notin \sigma \cup\{0\} .
$$

There is enough freedom in the choice of $w$ that we may assume that $(0, w)$ induces a triangulation $\Delta_{(0, w)}$ of $\rho(A)$ and not merely a subdivision. This also implies that $w$ can be chosen a weight vector for $H_{A}(\beta)$, if $\beta \in \mathbb{C}^{d}$ is given.

We claim that $(0, w) \cdot B \in \mathscr{K}_{\sigma}^{\circ}$. This implies that $\sigma$ is a maximal simplex in $\Delta_{(0, w)}$ which does not pass through zero. To prove the claim, note that

$$
\sum_{i \in \sigma}\left(w_{i}+\lambda_{0}\right) b_{i}+\sum_{i \notin \sigma \cup\{0\}}\left(w_{i}+\lambda_{0}-\lambda_{i}\right) b_{i}=\sum_{i=1}^{n}\left(h \cdot a_{i}\right) b_{i}=0 .
$$

Then

$$
\sum_{i=1}^{n} w_{i} b_{i}=\sum_{i \notin \sigma \cup\{0\}}\left(\lambda_{i}-\lambda_{0}\right) b_{i}-\lambda_{0} \sum_{i \in \sigma} b_{i}=\sum_{i \notin \sigma \cup\{0\}} \lambda_{i} b_{i}-\lambda_{0} \sum_{i=1}^{n} b_{i}=\sum_{i \notin \sigma} \lambda_{i} b_{i},
$$

where the last equality follows from $-b_{0}=\sum_{i=1}^{n} b_{i}$. But then, $\lambda_{i}>0$ for $i \notin \sigma$ implies that $(0, w) \cdot B \in \mathscr{K}_{\sigma}^{\circ}$, which is what we wanted.

The hypothesis that the columns of $A$ span a strongly convex cone cannot be removed from Lemma 7.2, as the following example shows.

Example 7.3. Let $A=\left[\begin{array}{lll}-1 & 1\end{array}\right]$. Then

$$
\rho(A)=\left[\begin{array}{rrr}
1 & 1 & 1 \\
0 & -1 & 1
\end{array}\right], \quad \text { and choose } B=\left[\begin{array}{r}
-2 \\
1 \\
1
\end{array}\right] .
$$

There are only two coherent triangulations of $\rho(A)$, namely

$$
\Delta_{1}=\{\{-1,1\}\} \text { and } \Delta_{2}=\{\{-1,0\},\{0,1\}\} .
$$

Their corresponding cones in the secondary fan are $\mathscr{K}_{\Delta_{1}}=\mathbb{R}_{\leq 0}$ and $\mathscr{K}_{\Delta_{2}}=\mathbb{R}_{\geq 0}$. For any vector $w=\left(w_{-1}, w_{1}\right) \in \mathbb{R}_{>0}^{2}$, the number

$$
0 \cdot b_{0}+w_{-1} \cdot b_{-1}+w_{1} \cdot b_{1}=w_{-1}+w_{1}
$$

belongs to the cone $\mathscr{K}_{\Delta_{2}}^{\circ}=\mathbb{R}_{>0}$ and consequently $w$ always induces a triangulation of $\rho(A)$ all of whose maximal simplices pass through zero.

Proposition 7.4. Assume that the columns of A span a strongly convex cone. If the rational rowspan of $A$ does not contain the vector $(1, \ldots, 1)$, there exists a weight vector $w$ such that the linear map

$$
\rho: \mathcal{N}_{w}\left(H_{A}(\beta)\right) \rightarrow \mathscr{N}_{(0, w)}\left(H_{\rho(A)}\left(\beta_{0}, \beta\right)\right)
$$

is not surjective, where $\beta_{0}$ is a homogenizing value for $A, \beta$ and $w$. 
Proof. Use Lemma 7.2 to pick $w$ so that the triangulation $\Delta_{(0, w)}$ of $\rho(A)$ has a maximal simplex that does not pass through zero. Then $\operatorname{in}_{(0, w)}\left(I_{\rho(A)}\right)$ has topdimensional standard pairs that do not pass through zero. Choose a homogenizing value $\beta_{0}$ for $A, \beta$ and $w$, and let $\left(v_{0}, v\right)$ be a fake exponent of $H_{\rho(A)}\left(\beta_{0}, \beta\right)$ corresponding to such a standard pair (fake exponents associated to top-dimensional standard pairs always exist). In particular, $v_{0} \in \mathbb{N}$. If $\left(v_{0}, v\right)$ has minimal negative support, it is an exponent of $H_{\rho(A)}\left(\beta_{0}, \beta\right)$ corresponding to a standard pair that does not pass through zero, and the associated logarithm-free solution $\phi_{\left(v_{0}, v\right)}$ of $H_{\rho(A)}\left(\beta_{0}, \beta\right)$ cannot belong to the image of $\rho$ by Theorem 3.18. If $\left(v_{0}, v\right)$ does not have minimal negative support, the argument of Proposition 3.4.16 in [24] produces an element $\left(v_{0}^{\prime}, v^{\prime}\right) \in\left(\left(v_{0}, v\right)+\operatorname{ker}_{\mathbb{Z}}(A)\right)$ whose negative support is minimal and strictly contained in the negative support of $\left(v_{0}, v\right)$, so that $v_{0}^{\prime}$ is still a non negative integer. Thus, the standard pair corresponding to $\left(v_{0}^{\prime}, v_{0}\right)$ cannot pass through zero, and $\phi_{\left(v_{0}^{\prime}, v^{\prime}\right)}$ is not in the image of $\rho$.

The following result is due to Berkesch (see Theorem 7.3 in [4]).

Theorem 7.5. If the cone over the columns of $A$ is strongly convex and $\beta_{0}$ is generic,

$$
\operatorname{rank}\left(H_{A}(\beta)\right)=\operatorname{rank}\left(H_{\rho(A)}\left(\beta_{0}, \beta\right)\right) .
$$

We now give a proof for Theorem 6.4.

Proof of Theorem 6.4. Given $\beta_{0}$ a homogenizing value for $A, \beta$ and $w$, Proposition 7.1 states that the spaces $\left.\mathcal{N}_{w}\left(H_{A}(\beta)\right)\right)$ and $\left.\mathscr{N}_{(0, w)}\left(H_{\rho(A)}\left(\beta_{0}, \beta\right)\right)\right)$ are isomorphic. We may further assume that $\beta_{0}$ is sufficiently generic that Theorem 7.5 holds.

As $I_{\rho}(A)$ is homogeneous, $H_{\rho(A)}\left(\beta_{0}, \beta\right)$ is regular holonomic, and Corollary 2.4.16 in [24] implies that $\operatorname{dim}_{\mathbb{C}}\left(\mathscr{N}_{(0, w)}\left(H_{\rho(A)}\left(\beta_{0}, \beta\right)\right)\right)=\operatorname{rank}\left(H_{\rho(A)}\left(\beta_{0}, \beta\right)\right)$. So,

$$
\begin{aligned}
\left.\operatorname{dim}_{\mathbb{C}}\left(\mathcal{N}_{w}\left(H_{A}(\beta)\right)\right)\right) & \left.=\operatorname{dim}_{\mathbb{C}}\left(\mathscr{N}_{(0, w)}\left(H_{\rho(A)}\left(\beta_{0}, \beta\right)\right)\right)\right) \\
& =\operatorname{rank}\left(H_{\rho(A)}\left(\beta_{0}, \beta\right)\right)=\operatorname{rank}\left(H_{A}(\beta)\right),
\end{aligned}
$$

where the last equality is by Theorem 7.5.

Since $w$ is a perturbation of $(1, \ldots, 1)$, if $\phi$ is a basic Nilsson solution of $H_{A}(\beta)$ in the direction of $w$, then $|u| \geq 0$ for all $u \in \operatorname{supp}(\phi)$. Therefore we can use Theorem 6.2 to find $\varepsilon \in \mathbb{R}_{>0}^{n-d}$ such that all basic Nilsson solutions of $H_{A}(\beta)$ in the direction of $w$ converge on $\mathscr{U}_{w, \varepsilon}$.

We are finally ready to show that, in the case when the columns of $A$ span a strongly convex cone, non-homogeneous $A$-hypergeometric systems are irregular for all $\beta$, thus generalizing the argument in Theorem 2.4.11 from [24], and providing an alternative proof of Corollary 3.16 from [25].

Theorem 7.6. Assume that the columns of $A$ span a strongly convex cone and $I_{A}$ is not homogeneous. Then $H_{A}(\beta)$ is not regular holonomic for any $\beta \in \mathbb{C}^{d}$. 
Proof. Choose $w$ a weight vector for $H_{A}(\beta)$ as in Proposition 7.4 and $\beta_{0}$ a homogenizing value for $A, \beta$ and $w$. Then

$$
\begin{aligned}
\operatorname{dim}_{\mathbb{C}}\left(\mathcal{N}_{w}\left(H_{A}(\beta)\right)\right) & <\operatorname{dim}_{\mathbb{C}}\left(\mathscr{N}_{(0, w)}\left(H_{\rho(A)}\left(\beta_{0}, \beta\right)\right)\right) \\
& =\operatorname{rank}\left(H_{\rho(A)}\left(\beta_{0}, \beta\right)\right) \\
& =\operatorname{rank}\left(H_{A}(\beta)\right) .
\end{aligned}
$$

The equality in the second line follows from Corollary 2.4.16 in [24] because the system $H_{\rho(A)}\left(\beta_{0}, \beta\right)$ is regular holonomic, as $I_{\rho(A)}$ is homogeneous. The last equality is by Theorem 7.5 (we may have to make $\beta_{0}$ more generic for this result to hold, but this does not affect Proposition 7.4). Now Corollary 2.4.16 in [24] and $\operatorname{dim}_{\mathbb{C}}\left(\mathcal{N}_{w}\left(H_{A}(\beta)\right)\right)<\operatorname{rank}\left(H_{A}(\beta)\right)$ imply that $H_{A}(\beta)$ is not regular holonomic.

\section{References}

[1] Adolphson, A.: Hypergeometric functions and rings generated by monomials. Duke Math. J. 73 (1994), no. 2, 269-290.

[2] Assi, A., Castro-Jiménez, F. J. And Granger, J. M.: How to calculate the slopes of a $\mathscr{D}$-module. Compositio Math. 104 (1996), no. 2, 107-123.

[3] Assi, A., Castro-Jiménez, F. J. and Granger, J. M.: The Gröbner fan of an $A_{n}$-module. J. Pure Appl. Algebra 150 (2000), no. 1, 27-39.

[4] Berkesch, C.: Euler-Koszul methods in algebra and geometry. PhD thesis, Purdue University, 2010.

[5] BJöRK, J.-E.: Rings of differential operators. North-Holland Mathematical Library, 21. North-Holland, Amsterdam-New York, 1979.

[6] Castro-Jiménez, F. J. and Fernández-Fernández, M. C.: Gevrey solutions of the irregular hypergeometric system associated with an affine monomial curve. Trans. Amer. Math. Soc. 363 (2011), no. 2, 923-948.

[7] Castro-Jiménez, F. J. And TAkayama, N.: Singularities of the hypergeometric system associated with a monomial curve. Trans. Amer. Math. Soc. 355 (2003), no. $9,3761-3775$.

[8] Cattani, E., Dickenstein, A. And Rodríguez Villegas, F.: The structure of bivariate rational hypergeometric functions. Int. Math. Res. Not. IMRN 2011 (2011), no. 11, 2496-2533.

[9] Cope, F. T.: Formal solutions of irregular linear differential equations. Part I. Amer. J. Math. 56 (1934), no. 1-4, 411-437.

[10] Fernández-Fernández, M. C.: Irregular hypergeometric $\mathscr{D}$-modules. Adv. Math. 224 (2010), no. 5, 1735-1764.

[11] Gel'fand, I. M., Graev, M.I. and Zelevinsky, A. V.: Holonomic systems of equations and series of hypergeometric type. Dokl. Akad. Nauk SSSR 295 (1987), no. 1, 14-19; translation in Soviet Math. Dokl. 36 (1988), no. 1, 5-10.

[12] Gel'fand, I. M., Kapranov, M.M. And Zelevinsky, A. V.: Discriminants, resultants, and multidimensional determinants. Mathematics: Theory \& Applications. Birkhäuser, Boston, MA, 1994.

[13] Gel'Fand, I. M., Zelevinsky, A. V. And Kapranov, M. M.: Hypergeometric functions and toric varieties. Funktsional. Anal. i Prilozhen., 23 (1989), no. 2, 12-26; translation in Funct. Anal. Appl. 23 (1989), no. 2, 94-106. 
[14] Hartillo Hermoso, M. I.: Slopes of hypergeometric systems of codimension one. In "Proceedings of the International Conference on Algebraic Geometry and Singularities (Sevilla, 2001)". Rev. Mat. Iberoamericana 19 (2003), no. 2, 455-466.

[15] Hartillo-Hermoso, M. I.: Irregular hypergeometric systems associated with a singular monomial curve. Trans. Amer. Math. Soc. 357 (2005), no. 11, 4633-4646.

[16] HotтA, R.: Equivariant D-modules. In Proceedings of ICPAM Spring School in Wuhan, 1991. Available at: arXiv:math/9805021v1.

[17] Laurent, Y.: Polygône de Newton et $b$-fonctions pour les modules microdifférentiels. Ann. Sci. École Norm. Sup. (4) 20 (1987), no. 3, 391-441.

[18] Laurent, Y. and Mebrhout, Z.: Pentes algébriques et pentes analytiques d'un D-module. Ann. Sci. École Norm. Sup. (4) 32 (1999), no. 1, 39-69.

[19] Matusevich, L.F., Miller, E. and Walther, U.: Homological methods for hypergeometric families. J. Amer. Math. Soc. 18 (2005), no. 4, 919-941.

[20] Mebkhout, Z.: Le théorème de comparaison entre cohomologies de de Rham d'une variété algébrique complexe et le théorème d'existence de Riemann. Inst. Hautes Études Sci. Publ. Math. 69 (1989), 47-89.

[21] Mora, T. and Robbiano, L.: The Gröbner fan of an ideal. J. Symbolic Comput. 6 (1988), no. 2-3, 183-208.

[22] Ohara, K. and Takayama, N.: Holonomic rank of $\mathscr{A}$-hypergeometric differentialdifference equations. J. Pure Appl. Algebra 213 (2009), no. 8, 1536-1544.

[23] Saito, M.: Logarithm-free A-hypergeometric series. Duke Math. J. 115 (2002), no. $1,53-73$.

[24] Saito, M., Sturmfels, B. And Takayama, N.: Gröbner deformations of hypergeometric differential equations. Algorithms and Computation in Mathematics, 6. Springer-Verlag, Berlin, 2000.

[25] Schulze, M. and Walther, U.: Irregularity of hypergeometric systems via slopes along coordinate subspaces. Duke Math. J. 142 (2008), no. 3, 465-509.

[26] Sturmfels, B.: Gröbner bases and convex polytopes. University Lecture Series, 8. American Mathematical Society, Providence, RI, 1996.

[27] Sturmfels, B., Trung, N. V. And Vogel, W.: Bounds on degrees of projective schemes. Math. Ann. 302 (1995), no. 3, 417-432.

Received July 23, 2010.

Alicia Dickenstein and Federico N. Martínez: Departamento de Matemática, FCEN, Universidad de Buenos Aires, C1428EGA Buenos Aires, Argentina.

E-mail: alidick@dm.uba.ar, fnmartin@dm.uba.ar

Laura Felicia Matusevich: Department of Mathematics, Texas A\&M University, College Station, TX 77843-3368, USA.

E-mail: laura@math.tamu.edu

Alicia Dickenstein was partially supported by UBACYT 20020100100242, CONICET PIP 112-200801-00483 and ANPCyT PICT 2008-0902, Argentina. Federico Nicolás Martínez was supported by a doctoral grant from CONICET. Laura Felicia Matusevich was partially supported by NSF Grant DMS 0703866 and a Sloan Research Fellowship. 